\title{
Precise, 3-D optogenetic control of the diameter of single arterioles
}

\author{
Philip J. O’Herron ${ }^{1,8, \#}$, David A. Hartmann"2,8, Kun Xie' ${ }^{1}$, Prakash Kara ${ }^{3,4,8}$, Andy Y. Shih ${ }^{5,6,7,8}$ \\ ${ }^{1}$ Department of Physiology, Augusta University, Augusta, Georgia, USA. \\ ${ }^{2}$ Department of Neurology \& Neurological Sciences, Stanford University, Stanford, California, USA. \\ ${ }^{3}$ Department of Neuroscience, University of Minnesota, Minneapolis, Minnesota, USA. \\ ${ }^{4}$ Center for Magnetic Resonance Research, University of Minnesota, Minneapolis, Minnesota, USA. \\ ${ }^{5}$ Center for Developmental Biology and Regenerative Medicine, Seattle Children's Research Institute, Seattle, \\ Washington, USA. \\ ${ }^{6}$ Department of Pediatrics, University of Washington, Seattle, Washington, USA. \\ ${ }^{7}$ Department of Bioengineering, University of Washington, Seattle, Washington, USA. \\ ${ }^{8}$ Department of Neuroscience, Medical University of South Carolina, Charleston, South Carolina, USA. \\ \# Corresponding author: poherron@augusta.edu
}

\section{Abstract}

Modulation of brain arteriole diameter is critical for maintenance of cerebral blood pressure and control of hyperemia during regional neural activity. However, studies of hemodynamic function in health and disease have lacked a method to control and monitor blood flow with high spatiotemporal resolution. Here, we describe a new all-optical approach to precisely control and monitor arteriolar contractility in vivo using combined two-photon optogenetics and imaging. The expression of the excitatory opsin, ReaChR, in vascular smooth muscle cells enabled rapid and repeated vasoconstriction following brief light pulses. Targeted two-photon activation of ReaCHR using a spatial light modulator (SLM) produced highly localized constrictions when targeted to individual arteries within the neocortex. We demonstrate the utility of this method for examining arteriole contractile dynamics and creating transient blood flow reductions. Additionally, we show that optogenetic constriction can offset or completely block sensory stimulus evoked vasodilation, providing a valuable tool to dissociate blood flow changes from neural activity.

\section{Introduction}

The brain is a metabolically demanding organ, consuming $20 \%$ of the body's energy supply despite being only $2 \%$ of our body weight ${ }^{1,2}$. To ensure an adequate supply of nutrients, cerebral vessels deliver blood where it is needed by dilating in response to local increases in neural activity - a process called functional hyperemia ${ }^{3}$. This coupling between neural and 
vascular activity is critical to healthy brain function, as deterioration of neurovascular coupling and impaired cerebral perfusion are seen in many cognitive disorders ${ }^{4-8}$. Additionally, the anatomical precision of the hyperemic response allows widely used imaging techniques such as functional Magnetic Resonance Imaging (fMRI) to use vascular signals to map regional neural activity on a scale of hundreds of micrometers ${ }^{9-13}$. Thus, there is a critical need for an accurate understanding of how neural activity interacts with and shapes hemodynamic responses.

One valuable approach in understanding the relationship between neural and vascular activity has been to manipulate blood vessels independently of local neural activity. Typically, studies have tried to alter cerebral arteriole diameter and blood flow using pharmacological or systemic manipulations (such as a $\mathrm{CO}_{2}$ challenge or system-wide changes in blood pressure) ${ }^{14-23}$. However, these methods do not provide the spatiotemporal specificity needed for functional hyperemia studies. They also exert broad systemic effects that make their impact on regional vascular diameter changes difficult to isolate. To improve the spatiotemporal control of blood flow, a few studies have optically controlled mural cell membrane potential using optogenetics, a technique initially developed for manipulating neural activity. These studies have typically expressed channelrhodopsin-2 (ChR2) in vascular mural cells, and activated ChR2 with blue light to constrict vessels. However, wide-field illumination with visible light limits the spatial resolution achievable, and predominantly activates the superficial vasculature ${ }^{24-26}$. Some studies have sought to improve the spatial resolution by using visible wavelength lasers to create a focal point of activation, but again, stimulation is limited to superficial tissue ${ }^{27-29}$. Achieving greater imaging depths has been attempted with fiber bundle probes ${ }^{30}$ but these have poor imaging quality and cause tissue damage. Two-photon activation allows deeper imaging and more precise spatial control than single-photon activation. This method has been used by us and others to activate ChR2 in mural cells to examine contractility with single-vessel resolution ${ }^{28,31}$. Recent work has also shown that two-photon activation of intravenous fluorescent dyes can produce local fluorescence for efficient one-photon activation of ChR2 in vascular smooth muscle ${ }^{32}$. However, the imaging and excitation in all of these studies were performed with the same laser, limiting the ability to spatiotemporally distinguish regions of stimulation from observation. Further, these studies used the original ChR2(H134R) variant, and opsins with improved two-photon cross sections have since become available.

Here, we introduce an experimental protocol to constrict cerebral arteries with high spatiotemporal resolution up to hundreds of micrometers below the surface. We use the redshifted opsin ReaChR ${ }^{33}$ which has strong photocurrents and a long activation wavelength well 
suited for two-photon stimulation. This method allows for constriction of individual or multiple branches of surface arteries or penetrating arterioles by driving cell-specific depolarization of smooth muscle cells. By combining dual-light paths and independent focusing of the excitation and imaging lasers, we constricted vessels independently from the depth of the imaging plane, making this a useful tool to manipulate and monitor vessel diameter in three dimensions. This approach is advantageous over ChR2 activation of vascular smooth muscle because it can manipulate single vessels over larger cortical depths, and longer wavelengths of light are less likely to be photo-toxic or create spurious vascular changes independent of opsin expression ${ }^{34}$.

\section{$\underline{\text { Results }}$}

The red-shifted opsin, ReaChR, potently depolarizes cells when illuminated with red light, ${ }^{33}$ and exhibits peak two-photon activation efficiency $\sim 1000 \mathrm{~nm} .{ }^{35}$ This makes it ideal for combined twophoton activation/imaging experiments, similar to the all-optical methods developed to activate neurons in vivo using long-wavelength light $(\sim 1040 \mathrm{~nm})$ while simultaneously imaging with shorter-wavelengths ( $800-950 \mathrm{~nm}){ }^{36-40}$. To selectively express ReaChR in mural cells, we crossed ReaChR reporter mice with PDGFRß-Cre mice (see Methods; Figure 1A), which allows expression of ReaChR tagged with mCitrine in nearly all vascular smooth muscle cells and pericytes, with negligible off-target expression in other cell types ${ }^{41}$ (Figure 1B). We imaged vasoconstrictive responses with two-photon microscopy while depolarizing the vascular mural cells either with a high power, pulsed $1040 \mathrm{~nm}$ laser or using a light-emitting diode (LED) for single-photon activation (Figure 1C).

\section{Single-photon activation of ReaChR in vessel walls}

We first examined ReaChR activation with single-photon stimulation using a $594 \mathrm{~nm}$ LED to stimulate vessels broadly across the cranial window. We found that a single $100 \mathrm{~ms}$ pulse of light led to the rapid and widespread constriction of pial arteries, which then relaxed back to baseline values within 30 seconds of activation (Figure 2A-C; Movie 1). This robust constriction ( $\sim 20 \%$ constriction from baseline level) to such a brief light stimulation is in stark contrast to activation with ChR2, where sustained stimulation over seconds were require for slow constrictions to appear ${ }^{28,31,32}$. In addition to the arterial constriction, we also observed a weaker constriction ( 2-3\%) in the surface veins (Figure 2A-C). Although some expression of the opsin was seen in venous mural cells (Figure 1B), the venous response was likely a passive deflation due to widespread reduction in blood flow caused by arteriole constriction ${ }^{14}$. Venous constriction was slower than the arterial constriction, with the time to $25 \%$ and full constriction ( 1.2 and 5.2 seconds respectively) delayed relative to arteries ( 0.6 and 3.3 seconds 
respectively). Additionally, targeted two-photon activation (described in detail below) produced large constrictions in arteries but not veins (Figure 2C).

We also observed that penetrating arterioles at least $300 \mu \mathrm{m}$ deep in the cortex constrict with single-photon activation (Figure 2D-E, Movie 2). When we applied repeated trains of $100 \mathrm{~ms}$ pulses, we were able to sustain penetrating arteriole constriction at $10-20 \%$ below baseline levels for over a minute while simultaneously imaging in between each pulse train (Figure 2F and Movie 3). To determine whether constricted arterioles reduced downstream microvascular flow, we performed a line scan on a penetrating arteriole and a first order pre-capillary branch $85 \mu \mathrm{m}$ deep in the tissue (Figure 2G). The number of red blood cells (seen as dark streaks in the line scan) was greatly reduced in the branch within one second of stimulation and flow completely stopped for approximately 2 seconds before returning to normal by 6.5 seconds post-stimulation (Figure $\mathbf{2 H}$ ). The penetrating arteriole rapidly constricted and the flow also stopped briefly around 2 seconds post-stimulation (Figure $2 \mathrm{H}$ ). Together, these results show that single photon activation of ReaChR exhibits rapid and robust constriction of cerebral arteries and reduced flow to the parenchymal tissue.

\section{Two-photon activation of ReaChR in vessel walls}

While single-photon LED activation leads to widespread vasoconstriction, it is not suitable for creating a focal point of activation for manipulation of individual vessels. Previous studies have used single-photon lasers focused through high numerical aperture (NA) objectives to target individual vessels in superficial tissue ${ }^{27-29,31}$. However, two-photon light provides greater resolution and allows the targeting of deeper vessels. Therefore, to investigate the spatial control achievable with two-photon optogenetic activation of arteries, we used a spatial light modulator (SLM) ${ }^{42,43}$ to split a single $1040 \mathrm{~nm}$ stimulation beam into multiple beamlets that could be focused on specific locations of interest. With this method, vasoconstriction was only observed when the stimulation spots were directly contacting the vessel wall (Figure 3A-E). No vasoconstriction was observed when stimulation spots were only $10 \mu \mathrm{m}$ from the vessel wall, indicating a high degree of spatial specificity in the $X-Y$ plane. The axial spread of activation, however, was much greater. Using the same objective (Nikon 16x 0.8 Numerical Aperture (N.A.)), we found the out-of-focus activation was strong up to at least $200 \mu \mathrm{m}$ above and below the focal plane (Figure 3F-G, Figure 3-1). Interestingly, the activation was strongest just outside the focal plane. This was also seen in a study of optogenetic activation of neurons which found that, at typical two-photon laser powers, photo-activation outside the focal plane can exceed that in the focal plane ${ }^{44}$. The explanation provided was that when laser power was high 
enough to saturate the opsins in the focal plane, the greater spread of the light beam outside the plane activated opsin molecules over a larger region than within the focal plane. However, using an objective with a higher numerical aperture (Nikon 25x 1.1 N.A.), we found that the activation was strongest at the focal plane compared to $50 \mu \mathrm{m}$ away and by $150 \mu \mathrm{m}$ outside the focal plane, the activation had nearly disappeared (Figure 3G). Although this spread of activation will limit the ability to constrict small sections of a penetrating arteriole, it could be advantageous when trying to reduce flow to the entire region of cortex perfused by a single arteriole (see below). In addition to the objective optics, we expect that the amount of out-offocus activation will also depend on the laser power applied, the orientation of the vessel relative to the light cone, and the size of the arteriole. Thus, care must be taken in the experimental design to avoid unwanted activation of vessels located within the light cone but outside the focal plane.

When we placed stimulation spots on pial arteries, we observed robust constriction that was spatially constrained to a few tens of micrometers around the stimulation spots. Indeed, we were able to constrict neighboring branches of a surface artery, separated by less than $300 \mu \mathrm{m}$ of vascular distance, completely independently from each other with no overlap of constrictive response (Figure 3H-L, Movies 4\&5). Increasing the laser power did not lead to widespread propagation of the constriction. A 30-fold increase in total power (5 to $150 \mathrm{~mW}$ ) led to a doubling in the magnitude of constriction at the stimulation site (from $\sim 5 \%$ to $\sim 10 \%$ ) and a $\sim 3$-fold increase in spread of constriction (from $25 \mu \mathrm{m}$ away from the center of stimulation to $75 \mu \mathrm{m}$ )

(Figure 4A-H). When we further increased the power to $\sim 225 \mathrm{~mW}$, the vessel wall was damaged causing the i.v. dye to leak out. Although the threshold for damage varied depending on vessel size and the number and arrangement of the stimulation spots, we regularly observed that excessively high powers (typically $>200 \mathrm{~mW}$ total) on pial arteries caused dye to leak out of the vessel walls and occasionally this was accompanied by widespread constriction covering most or all of the field of view (Movie 6).

\section{Control for laser light/heat}

Light has been shown to modify vessel diameter in the absence of opsin ${ }^{26,34}$. Therefore, to ensure that the light and/or the heat from the laser was not affecting our measurements, we used control mice expressing fluorophore (YFP) but no opsin in the vascular smooth muscle cells. These mice did not show focal constriction at any power level (Figure 4I-L; Movies 7 \& 9). Occasionally, at very high laser powers (>300 $\mathrm{mW}$ total power), we observed the same widespread constriction and vessel damage seen in the opsin animals (Movies 8 \& 10). 
Therefore, the focal constrictions produced by $1040 \mathrm{~nm}$ two-photon absorption below $150 \mathrm{~mW}$ are ReaChR-dependent.

\section{Single-vessel precision at depth}

Two-photon stimulation also allows precise targeting of vessels below the cortical surface. We targeted a region with three penetrating arterioles $170 \mu \mathrm{m}$ below the cortical surface. We were able to constrict all three arterioles simultaneously by splitting the stimulation beam and positioning spots over each vessel (Figure 5A-B). Alternatively, we could also stimulate just a single vessel at a time (Figure $\mathbf{5 C}$ ), showing precise control of vascular diameter between neighboring penetrating arterioles.

The SLM also allowed us to dissociate the imaging and stimulation focal planes. We leveraged this capacity to study the spread of optogenetically-induced constriction in penetrating arterioles by stimulating $170 \mu \mathrm{m}$ below the cortical surface and imaging both at the cortical surface and $300 \mu \mathrm{m}$ below the surface. We found robust constrictions across the entire observed span of the penetrating arteriole (Figure 5D-F). In contrast, stimulation of a surface vessel led to more spatially constrained constrictions in the lateral plane of the vessel $(<50 \mu \mathrm{m}$; Figure $\mathbf{5 G})$, as seen above (Figures 3-4). This discrepancy in the spread of constriction between pial and penetrating artery activation is in part due to out-of-focus excitation in the axial plane (see Figure 3F-G, Figure 3-1). However, some of the spread may be due to greater conductance of constriction along the penetrating arteriole. When considering the axial point-spread function of optogenetic activation, we detected minimal constriction of surface vessel when the focal plane was placed 150-200 $\mu \mathrm{m}$ below the surface (Figure 3G). In contrast, during stimulation of a penetrating arteriole, we saw robust constrictions of surface arterioles when the focal plane of stimulation was $170 \mu \mathrm{m}$ below, indicating potential upstream conduction of the constrictive response. Thus, it is possible that penetrating arterioles respond to the optogenetic activation differently from pial arteries. Regardless of the mechanism of the widespread constriction, since penetrating arterioles supply columns of underlying cortical microvasculature ${ }^{45}$, this finding shows that blood flow to discrete regions of cortex can be reversibly and spatially modulated by optogenetic contraction of penetrating arterioles.

\section{Comparison of contractile kinetics with sensory-evoked vasodilation}

One of the hallmarks of neurovascular coupling is the long delay between the onset of neural activity and vascular responses ${ }^{46}$. In primary sensory areas, neurons typically respond a few tens of milliseconds after stimulus onset. However, vasodilation typically takes more than 500 
ms to begin and may not peak for 5 or more seconds following stimulus onset, explaining the limited temporal precision achievable with FRRI $^{46,47}$. Using optogenetic stimulation to directly activate smooth muscle cells, we find that the vasoconstriction occurs very rapidly - typically the vessels were constricting by the first imaging frame following stimulation onset. To directly compare the kinetics of optogenetically-evoked constriction with sensory-evoked dilation in the same vessel, we presented visual stimuli and measured dilation of a penetrating arteriole, and then subsequently stimulated the same arteriole with $1040 \mathrm{~nm}$ light. As expected, optogenetic constriction of the arteriole showed a much shorter onset ( $25 \%$ to peak constriction: 0.60 seconds) than the visually evoked dilation ( $25 \%$ to peak dilation: 3.4 seconds; Figure $6 \mathrm{~A}$ ). To more accurately gauge the onset time of the optogenetically-evoked constriction, we activated a set of vessels during high speed $(15 \mathrm{~Hz})$ imaging. We fit the responses with a constant (for the baseline interval) followed by an exponential (for the initial response phase) and estimated the constriction onset latency to be 266 ms (95\% Cl: 109-423 ms; Figure 6B).

In addition to the rapid onset, the rate of the diameter change was also faster with optogenetic constriction ( 0.73 seconds from $25 \%-75 \%$ peak) than sensory-evoked dilation (1.90 seconds from $25 \%-75 \%$ peak). However, despite a 50 -fold difference in stimulus duration (100 ms of $1040 \mathrm{~nm}$ light versus 5 seconds of visual stimuli) and the more rapid response onset rate, the decay back to baseline was only marginally faster in the light-stimulation condition (8.4 seconds from peak constriction back to $25 \%$ peak vs. 10.9 seconds peak dilation back to $25 \%$ in sensory-evoked). Altogether, these studies indicate that smooth muscle cells are capable of changing vessel diameter much more rapidly than is suggested by the kinetics of sensoryevoked functional hyperemia.

\section{Using optogenetic stimulation to offset sensory-evoked dilation}

We then asked whether optogenetically-driven constriction could override vasodilation during neurovascular coupling, providing a means to dissociate neural activity from the vascular responses. We presented visual and optogenetic stimulation together and found that the vessel was rapidly constricted by the light pulse and then a dilation followed that was lower in amplitude than the dilation to the sensory stimulus alone (Figure 6C-D green versus blue). By reducing the visual stimulus duration from five seconds to two seconds, the same light pulse completely suppressed the dilation phase of the vascular response (Figure 6C-D red). Thus, with the right balance of timing and power of the stimulation laser, vasodilation to a sensory stimulus can be completely negated. This is a powerful tool to modulate vascular contributions 
to hyperemia during neurovascular coupling. For instance, it can be used to study the impact of blunted hyperemia on neural function, which is relevant to numerous diseases ${ }^{3,5}$.

\section{Discussion}

Borrowing from the techniques recently developed for all-optical interrogation of ensembles of neurons ${ }^{36,37,40}$, here we introduce a technique for all-optical interrogation of the vasculature. We expressed the red-shifted opsin ReaChR in vascular mural cells to rapidly and reversibly control blood flow over a range of spatiotemporal scales. Single-photon activation produced widespread vasoconstriction across the full cranial window ( 1-2 $\mathrm{mm})$, whereas two-photon activation induced constriction of individual vessels or branches in a very localized region (10s of micrometers). By utilizing a dual light path system and an SLM coupled to a pulsed 1040nm laser, we were able to control the diameter of individual vessel segments independently in three dimensions. This paradigm allowed us to investigate the spatial and temporal dynamics of arterial vasoconstriction in unprecedented detail. We demonstrate that single brief pulses of light (100 ms) produce robust vasoconstriction with rapid onset (<300 ms), which could be repeatedly administered to maintain prolonged constriction while simultaneously imaging the tissue. Activation of penetrating arterioles led to constriction along the span of the vessel more than 100 micrometers from the stimulation plane, suggesting they may exhibit different conduction properties than pial arterioles, which exhibited very focal constrictions. Altogether, we find that optical stimulation of mural cells expressing ReaChR is a powerful tool for studying cerebral vasoconstriction, and for dissecting neuronal and vascular activity across with high spatiotemporal precision.

Prior studies have utilized ChR2 to achieve vasoconstriction in vascular mural cells. In the living mouse brain, one- and two-photon activation of ChR2 led to the gradual constriction of smooth muscle cells, requiring many seconds of constant activation for an appreciable response ${ }^{28,31,32}$. Our finding that $100 \mathrm{~ms}$ of ReaChR illumination induces robust constriction in less than 1 second suggests that ReaChR is superior to ChR2 for two-photon optogenetic studies of the vasculature. This is likely because of the higher photocurrent amplitude in ReaChR compared to ChR2 (H134R variant) for one-photon activation at their respective optimum excitation wavelengths ${ }^{33}$, as well as the superior tissue penetration of longer-wavelength lights used to activate ReaChR ${ }^{35}$. ReaChR is among the most potent red-shifted opsins for two-photon activation as shown in neurons ${ }^{48}$, and it is more convenient for use in the vasculature than ChRmine ${ }^{40}, \mathrm{C} 1 \mathrm{~V} 1{ }^{49}$, or other red-shifted opsins because of its availability in Cre-dependent mouse lines. An additional advantage is that the optimal activation wavelength of ReaChR (970- 
$1040 \mathrm{~nm}$ ) does not substantially interfere with imaging of common fluorophores, whereas twophoton ChR2 activation occurs at the same imaging wavelengths as widely-used calcium indicators such as Oregon Green or GCaMP ${ }^{50}$. This feature of ReaChR permits independent imaging and stimulation with separate laser lines.

The highly localized constriction we see following a focal activation differs from vasodilation responses seen during functional hyperemia. Following focal activation of neural tissue, vasodilatory signals propagate several millimeters from the activation site ${ }^{51,52}$. This long-range propagation of dilation is attributed to endothelial cell signaling through gap junctions ${ }^{53-55}$, and influences the spatial precision of vascular imaging (e.g. fMRI) signals ${ }^{9,56}$. The lack of propagation seen here when activating smooth muscle cells supports the notion that endothelial rather than smooth muscle cells mediate the long-range propagation of signals through vessel walls. However, differences in the propagation characteristics of dilatory versus constrictive signals and in neural activation versus direct stimulation of vessel walls may also play a role in the propagation range of the signals.

Although the constriction signals in surface arteries were constrained to a few tens of micrometers from the activation site, we found that focal activation of penetrating arteries led to constriction $\sim 200 \mu \mathrm{m}$ away from the stimulation plane. This is likely due in part to the axial point-spread function and out-of-focus activation along the length of the vessel. However, the spread of constriction through penetrating arterioles appears greater than what we found in a control experiment, where a longitudinally oriented surface arteriole did not constrict appreciably when it was $150 \mu \mathrm{m}$ or more out of the focal plane of stimulation (compare Figure $\mathbf{3 G}$ with Figure 5E-F). Thus there may be real biological differences - such as astrocyte endfeet coverage on penetrating but not pial arteries, and differences in ion channel activity and myogenic tone between pial and penetrating arteries - that might lead to differences in propagation between surface and penetrating vessels ${ }^{57-60}$. In support of this possibility, a modelling study proposed that propagation of constriction along penetrating arterioles is necessary to explain experimentally observed flow/velocity decreases seen after constriction of these vessels with light ${ }^{61}$. Future studies stimulating penetrating vessels that take a non-vertical trajectory will help parse out any biological difference in propagation between pial and penetrating vessels.

Another similar question of interest is how signals propagate from capillaries back to the feeding arterioles. With SLM optogenetics, one could stimulate numerous capillary pericytes while measuring diameter changes in upstream penetrating arterioles. The ability of pericytes to 
communicate with upstream vessels to influence blood flow is appreciated in ex vivo retinal preparations ${ }^{62}$, but has yet to be shown in vivo in the brain. Indeed the role capillaries play in control of blood flow in the brain is a hotly debated topic in the field $22,28,63-66$. Optogenetic activation of mural cells with ChR2 was found to constrict arterioles but not capillaries in one study, ${ }^{28}$, but our group and others recently showed that prolonged stimulation of pericytes expressing ChR2(H134R) can lead to a slow constriction in capillaries ${ }^{29,31}$. The superior sensitivity of the ReaChR opsin, and the separation of imaging from stimulation with dual laser lines, will be useful tools for clarifying the ability of capillaries to control blood flow.

This technique can also be combined with different genetic strategies to probe other aspects of vascular wall function. For instance, using a different Cre line, ReaChR could be targeted to endothelial cells to monitor their response properties to light activation. Interestingly, despite the fact that increasing $\mathrm{Ca}^{2+}$ in endothelial cells (for example with Acetylcholine) leads to vasodilation ${ }^{67}$, photoactivation of these cells with ChR2 was shown to cause vasoconstriction 25. Another possibility is to insert hyperpolarizing opsins into smooth muscle or endothelial cells to study vasodilation ${ }^{27,68}$. Although the development of red-shifted hyperpolarizing opsins has lagged behind excitatory variants, promising candidates have recently emerged ${ }^{39,69-71}$.

Monitoring neural or astrocytic activity along with vessels during vascular manipulation opens up additional opportunities to study questions on neuro-glia-vascular coupling. For instance, offsetting stimulus evoked dilations (Figure 6C) while monitoring neural activity with high spatial precision can shed light on what aspects of the functional hyperemia response are essential to neuronal function. For instance, loss of vascular reactivity during functional hyperemia is seen in small vessel disease and other neuro-cognitive disorders ${ }^{4,5}$. Studies to optically suppress arteriole dilation may shed light on how this vascular dysfunction affects blood delivery and activity of neurons acutely during functional hyperemia. Furthermore, it has been shown that altering vessel tone can modify neural and astrocyte signals in vitro, so-called vasculo-neuronal coupling ${ }^{72}$. Establishing this property in vivo has been difficult due to technical limitations on the ability to control vessels without affecting other cell types. The direct activation of vessels that bypasses neuro-glia pathways provides a valuable technique to probe the mechanisms of this vasculo-neuronal coupling.

In summary, here we present a novel technique to directly manipulate vascular diameter in vivo. The rapid, robust, and reversible vasoconstriction we observed with ReaChR activation is unattainable with previous pharmacological or optogenetic techniques. With two-photon activation we were able to manipulate the vasculature with single-vessel resolution over 
hundreds of micrometers of cortical depth. The unprecedented spatiotemporal control of blood

flow conferred by this method and the ability to simultaneously monitor activity in the tissue

should make this a valuable technique to enhance our understanding of how vessels coordinate with other brain cells to fulfill the high energetic demands of the brain. 


\section{Materials and Methods}

Experiments were performed at the Medical University of South Carolina (MUSC) and Augusta University $(A U)$. All surgical and experimental procedures were approved by the Institutional Animal Care and Use Committees of the Universities.

Animals: Mice were generated at our Institutions by crossing heterozygous PDGFR $\beta$-Cre male mice (courtesy of Dr. Volkhard Lindner, Maine Medical Center Research Institute) with heterozygous female opsin mice (floxed ReaChR-mCitrine; Jackson Laboratories: Strain \#026294). Control mice were generated by crossing the same PDGFR $\beta$-Cre males with Ai3 females (floxed YFP; Jackson Laboratories: Strain \#007903). Mice inheriting the genes of interest from both parents displayed green fluorescence under blue illumination in tail snips or ear punches. Male and female mice were used aged 2-12 months.

Surgeries: Mice were anesthetized with a bolus infusion of fentanyl citrate $\left(0.04-0.05 \mathrm{mg} \mathrm{kg}^{-1}\right)$, midazolam (4-5 mg kg-1), and dexmedetomidine $\left(0.20-0.25 \mathrm{mg} \mathrm{kg}^{-1}\right)$. The heart and respiration rates of the animals were continually monitored throughout the surgeries. The scalp was excised, the skull cleaned, and a custom-made head-plate was fixed to the skull with C\&B MetaBond quick adhesive cement (Parkell; S380). Craniotomies (2-3 mm) were opened over the primary visual cortex centered approximately $2.5 \mathrm{~mm}$ lateral to the lambda suture and 1-1.5 $\mathrm{mm}$ anterior to the transverse sinus. Craniotomies were sealed with a specially-made glass coverslip consisting of a round $3 \mathrm{~mm}$ glass coverslip (Warner Instruments; 64-0720 (CS-3R)) glued to a round $4 \mathrm{~mm}$ coverslip (Warner Instruments; 64-0724 (CS-4R)) with UV-cured optical glue (Norland Products; 7110). The coverslip was positioned with the $3 \mathrm{~mm}$ side placed directly over the cortical surface, while the $4 \mathrm{~mm}$ coverslip laid on the skull at the edges of the craniotomy. An instant adhesive (Loctite Instant Adhesive 495) was carefully dispensed along the edge of the $4 \mathrm{~mm}$ coverslip to secure it to the skull, taking care not to allow any spillover onto the brain. Lastly, the area around the cranial window was sealed with dental cement. Animals were given at least 3 weeks to recover and to ensure the window would be optically clear before imaging took place.

Animal imaging: During imaging sessions, mice were anesthetized with $\sim 30 \mu \mathrm{L}$ chlorprothixene $(1 \mathrm{mg} / \mathrm{mL}$, intramuscular) and low levels of isoflurane $(\leq 0.8 \%)$. Heart rate and respiration rate were continuously monitored to ensure consistent anesthetic depth and animals were kept on a heating pad to maintain body temperature. Either fluorescein isothiocyanate (FITC) dextran (MW = $2000 \mathrm{kDa})$ or Texas Red $(70 \mathrm{kDa})$ dextran $(5 \% \mathrm{w} / \mathrm{v}$ in saline $)$ was injected 
retro-orbitally $(20-40 \mu \mathrm{L})$ under an initial high level of isoflurane $(\sim 2 \%)$. When visual stimuli were presented, the injections were in the eye not being stimulated by the computer display monitor. Isoflurane levels were returned to $\leq 0.8 \%$ for at least 15 minutes before data collection began.

Equipment: Imaging data was collected using the Ultima 2P-Plus two-photon microscope system (Bruker Corporation). At MUSC we used a beta version of the Ultima 2P-Plus. The major differences from the full version at $\mathrm{AU}$ were that the beta version did not have the $594 \mathrm{~nm}$ LED for single-photon stimulation or the Electro-Tunable Lens. So stimulation and imaging in different depths had to be performed with a Spatial Light Modulator (SLM) ${ }^{42,43}$. At MUSC, an Insight X2 (Spectra-Physics, MKS Instruments Inc.) was used for imaging and a FemtoTrain (Spectra-Physics: $1040 \mathrm{~nm}, \sim 3.5 \mathrm{~W}$ average power; < $370 \mathrm{fs}$ pulse width; > $350 \mathrm{~nJ}$ pulse energy) was used for optogenetic stimulation. At $A U$ we used the tunable line from the Insight X3 (Spectra-Physics) for imaging and the fixed $1045 \mathrm{~nm}$ line ( 4W average power; <170 fs pulse width; $\sim 44 \mathrm{~nJ}$ pulse energy) from the same laser for stimulation. Dedicated fixed wavelength lasers used for two-photon optogenetics typically have lower repetition rates and higher pulse powers than imaging lasers like the Insight X3. Nevertheless, despite the lower pulse power, the fixed line from the Insight was more than sufficient to evoke constrictions even deep in the cortex. Imaging was performed through a 25x (1.1 N.A., $2 \mathrm{~mm}$ Working Distance) or 16x (0.8 N.A., $3 \mathrm{~mm}$ WD) Nikon objectives. Results from each institution were similar, and therefore combined.

Because of the intensity of the stimulation light (for both single- and two-photon illumination) and the potential for extremely bright fluorescence from stimulation laser excitation, the PMT detectors were fitted with fast mechanical shutters which block incoming light during the optical stimulation periods. This is seen in all the movies as a brief dimming of the image when the stimulation light is on. Our strategy was to use brief stimulation intervals with long delays between them to allow sufficient imaging time to monitor arterial reaction to the stimulation.

The LED on the Ultima 2P-Plus was positioned in the light path on the collection side of the primary dichroic (Figure 1C). To send this light to the objective, a cube was positioned in the path between the PMT and the objective containing both a mirror that reflects a band of light around the LED wavelength $(570-605 \mathrm{~nm})$ and a filter that blocks red light from reaching the PMT detectors (near-zero transmission band from 575-605 nm). Thus imaging in the red channel was mostly lost when using the LED for stimulation. Therefore we used FITC dextran to image the vessels in this mode. 
Optical Stimulation: A. Single-photon. For single-photon stimulation, we used a 594 nm LED. We used $100 \mathrm{~ms}$ pulses of light. When a train of pulses was given, there were $100 \mathrm{~ms}$ between pulses. We used a single pulse (Figure 2A-C), a train of 5 pulses (Figure 2E), or a train of 10 pulses (Figure 2D). When we were trying to maintain constriction over a long duration (Figure 2F), we used repeated trains of 5 pulses separated by 4 seconds.

B. Two-photon. For two-photon stimulation, we typically had 4 or 6 spots presented at a time. They were spiraled over a small region $(12-25 \mu \mathrm{m})$ to increase the area of activation ${ }^{36,37}$. Single $100 \mathrm{~ms}$ pulses were nearly always used. Laser powers were typically 30-50 mW per spot for surface vessels and up to $90 \mathrm{~mW}$ per spot for penetrating arterioles. Although higher powers led to damage of vessel walls and dye leakage on the surface in pial arteries, even the highest powers we used in penetrating arterioles never led to visible damage or leakage from those vessels. To determine the power of the stimulation spots, we used a power meter while presenting random patterns of 4 and 6 spots. It is not feasible to calibrate the power in each spot separately so we divided the overall power by the number of spots to obtain an estimate of the power of each spot. The total power varied somewhat (typically $<10 \%$ ) from pattern to pattern and the amount of power each spot receives depends on its position on the SLM ${ }^{39}$. Since it was not possible to know what spot pattern was required until we were positioning them on the vessels, the exact power applied to each spot in each experiment is an estimated range.

Optical Imaging: Images were usually collected at 3.75 frames/second, except for Figure 6B (15 frames/second). Vessels were imaged at $875 \mathrm{~nm}$ when labeled with Texas Red dextran and $800 \mathrm{~nm}$ when imaged with FITC dextran.

Although $920 \mathrm{~nm}$ is in the tail of the activation spectrum for red-shifted opsins like ReaChR, wavelengths in this range are typically used in all-optical (activation and imaging) neural approaches $36,37,40$. These previous studies have reported that using low enough laser powers for imaging prevents photo-activation from the imaging laser. We typically imaged Texas Red at $875 \mathrm{~nm}$ and saw no evidence that the imaging laser led to vasoconstriction. When we imaged at $920 \mathrm{~nm}$, we did see slight constrictions at higher powers but were able to lower the power to avoid this effect while still obtaining adequate image quality (data not shown). The possibility of constriction due to the imaging laser is important to keep in mind when choosing an opsin/indicator combination. Although more sensitive opsins are usually desirable because less light is needed to activate them and the stimulation beam can be split into more beamlets, it will also make the tissue more readily activated by the imaging laser. 
It is also important to consider the effects of the emitted fluorescence on the opsin. Texas Red emission is at the excitation peak for single-photon activation of ReaChR. This feature was elegantly used in a recent study to greatly improve the spatial precision of single-photon excitation ${ }^{32,73}$. While we did not see evidence of a constant constriction during imaging caused by the emission of Texas Red (further constriction was always possible and vessels did not rapidly constrict at the start of an imaging run), the constriction seen with higher imaging powers that we attributed to imaging laser activation, may have at least in part been caused by the greater emission of photons from the Texas Red molecules passing through the imaging plane.

Visual Stimuli: Drifting square-wave grating stimuli were presented on a 17-inch LCD monitor. The gratings were presented at $100 \%$ contrast, $30 \mathrm{~cd} \mathrm{~m}^{-2}$ mean luminance, $1.5 \mathrm{~Hz}$ temporal frequency, and 0.04 cycles/degree. The visual stimulus and the optogenetic pulses were both controlled by the imaging software, ensuring that the onset of both stimulation types were synchronized.

Data Analysis: Data were analyzed in Matlab (Mathworks) and ImageJ (National Institutes of Health). Blood vessel diameters were computed frame-by-frame using custom Matlab scripts 9,74. Diameters were normalized by the average of 15 frames prior to stimulation onset. Repetitions were aligned by the onset of the optical stimulation when averaged together. The latency of constriction computed in Figure 6B was based on the time point where phase one of a fit equation switched to phase two (see ${ }^{75}$ ). The fit is given by:

$A \cdot$ phase one $(t)+\left[A+C\left(\exp \left(-\left(t-t_{1}\right) / \tau\right)-C\right] \cdot\right.$ phase two $(t)$

Where: phase one $(t)=\left\{\begin{array}{c}1 \text { for } t<t_{1} \\ 0 \text { else }\end{array}\right\}$ and phase two $(t)=\left\{\begin{array}{c}1 \text { for } t_{1} \leq t<2 \text { seconds } \\ 0 \text { else }\end{array}\right\}$

$A$ is a constant representing the baseline amplitude (nearly 1 ), $C$ is the amplitude of the exponential and $\mathrm{T}$ is its time constant. The time point to switch between the two phases is the parameter $t_{1}$, which gives the onset latency. The data to fit are cut off at 2 seconds which was determined by eye to be near the rising phase of the response. We also determined the latency by regressing the data on the constricting phase of the response. The $20 \%$ and $80 \%$ of the minimum of the curve were set as the bounds for the regression and a line was fit to this data. The point where this line crossed the average baseline value was used as the onset. However, the slope of this regression put the onset slightly before the stimulus onset so it was not deemed an accurate measure. 


\section{$\underline{\text { References: }}$}

1 Raichle, M. E. \& Gusnard, D. A. Appraising the brain's energy budget. Proc Natl Acad Sci U S A 99, 10237-10239 (2002).

2 Clark, D. \& Sokoloff, L. (Philadelphia: Lippincott, 1999).

3 ladecola, C. The Neurovascular Unit Coming of Age: A Journey through Neurovascular Coupling in Health and Disease. Neuron 96, 17-42 (2017).

4 ladecola, C. The pathobiology of vascular dementia. Neuron 80, 844-866 (2013).

5 Sweeney, M. D., Kisler, K., Montagne, A., Toga, A. W. \& Zlokovic, B. V. The role of brain vasculature in neurodegenerative disorders. Nat Neurosci 21, 1318-1331 (2018).

6 Gorelick, P. B. et al. Vascular contributions to cognitive impairment and dementia: a statement for healthcare professionals from the american heart association/american stroke association. Stroke 42, 2672-2713 (2011).

7 Snyder, H. M. et al. Vascular contributions to cognitive impairment and dementia including Alzheimer's disease. Alzheimers Dement 11, 710-717 (2015).

8 Lourenço, C. F., Ledo, A., Dias, C., Barbosa, R. M. \& Laranjinha, J. Neurovascular and neurometabolic derailment in aging and Alzheimer's disease. Frontiers in Aging Neuroscience 7 (2015).

9 O'Herron, P. et al. Neural correlates of single-vessel haemodynamic responses in vivo. Nature 534, 378-382 (2016).

10 Vanzetta, I., Slovin, H., Omer, D. B. \& Grinvald, A. Columnar resolution of blood volume and oximetry functional maps in the behaving monkey; implications for FMRI. Neuron 42, 843-854 (2004).

11 Vazquez, A. L., Fukuda, M., Crowley, J. C. \& Kim, S. G. Neural and hemodynamic responses elicited by forelimb- and photo-stimulation in channelrhodopsin-2 mice: insights into the hemodynamic point spread function. Cereb Cortex 24, 2908-2919 (2014).

12 Yacoub, E., Harel, N. \& Uğurbil, K. High-field fMRI unveils orientation columns in humans. Proceedings of the National Academy of Sciences 105, 10607-10612 (2008).

13 Fukuda, M. et al. Localization of Activity-dependent Changes in Blood Volume to Submillimeter-scale Functional Domains in Cat Visual Cortex. Cerebral Cortex 15, 823833 (2005).

14 Masamoto, K. \& Vazquez, A. Optical imaging and modulation of neurovascular responses. J Cereb Blood Flow Metab 38, 2057-2072 (2018).

15 Tarantini, S. et al. Pharmacologically-Induced Neurovascular Uncoupling is Associated with Cognitive Impairment in Mice. Journal of Cerebral Blood Flow \& Metabolism 35, 1871-1881 (2015).

16 Leithner, C. et al. Pharmacological uncoupling of activation induced increases in CBF and CMRO2. J Cereb Blood Flow Metab 30, 311-322 (2010).

17 Blinder, $\mathrm{P}$. et al. The cortical angiome: an interconnected vascular network with noncolumnar patterns of blood flow. Nature Neuroscience 16, 889-897 (2013). 
18 Hutchinson, E. B., Stefanovic, B., Koretsky, A. P. \& Silva, A. C. Spatial flow-volume dissociation of the cerebral microcirculatory response to mild hypercapnia. Neuroimage 32, 520-530 (2006).

19 Jenkins, B. G. Pharmacologic magnetic resonance imaging (phMRI): imaging drug action in the brain. Neuroimage 62, 1072-1085 (2012).

20 Suh, J. Y. et al. Reduced microvascular volume and hemispherically deficient vasoreactivity to hypercapnia in acute ischemia: MRI study using permanent middle cerebral artery occlusion rat model. J Cereb Blood Flow Metab 35, 1033-1043 (2015).

$21 \mathrm{Lu}, \mathrm{J}$. et al. Characterization of cerebrovascular responses to hyperoxia and hypercapnia using MRI in rat. Neuroimage 45, 1126-1134 (2009).

22 Grutzendler, J. \& Nedergaard, M. Cellular Control of Brain Capillary Blood Flow: In Vivo Imaging Veritas. Trends Neurosci 42, 528-536 (2019).

23 Nishimura, N., Rosidi, N. L., ladecola, C. \& Schaffer, C. B. Limitations of collateral flow after occlusion of a single cortical penetrating arteriole. J Cereb Blood Flow Metab 30, 1914-1927 (2010).

$24 \mathrm{Wu}, \mathrm{Y}$. et al. Optogenetic approach for functional assays of the cardiovascular system by light activation of the vascular smooth muscle. Vascul Pharmacol 71, 192-200 (2015).

25 Zhang, S. et al. Optogenetic intervention to the vascular endothelium. Vascul Pharmacol 74, 122-129 (2015).

26 Rorsman, N. J. G., Ta, C. M., Garnett, H., Swietach, P. \& Tammaro, P. Defining the ionic mechanisms of optogenetic control of vascular tone by channelrhodopsin-2. Br J Pharmacol 175, 2028-2045 (2018).

27 Mateo, C., Knutsen, P. M., Tsai, P. S., Shih, A. Y. \& Kleinfeld, D. Entrainment of Arteriole Vasomotor Fluctuations by Neural Activity Is a Basis of Blood-OxygenationLevel-Dependent "Resting-State" Connectivity. Neuron 96, 936-948.e933 (2017).

28 Hill, R. A. et al. Regional Blood Flow in the Normal and Ischemic Brain Is Controlled by Arteriolar Smooth Muscle Cell Contractility and Not by Capillary Pericytes. Neuron 87, 95-110 (2015).

29 Nelson, A. R. et al. Channelrhodopsin Excitation Contracts Brain Pericytes and Reduces Blood Flow in the Aging Mouse Brain in vivo. Frontiers in Aging Neuroscience 12 (2020).

30 Kim, M., Hong, J., Kim, J. \& Shin, H. J. Fiber bundle-based integrated platform for widefield fluorescence imaging and patterned optical stimulation for modulation of vasoconstriction in the deep brain of a living animal. Biomed Opt Express 8, 2781-2795 (2017).

31 Hartmann, D. A. et al. Brain capillary pericytes exert a substantial but slow influence on blood flow. bioRxiv, 2020.2003.2026.008763 (2020).

32 Tong, L. et al. Single cell in vivo brain optogenetic stimulation by two-photon excitation fluorescence transfer. bioRxiv, 2020.2006.2029.179077 (2020).

33 Lin, J. Y., Knutsen, P. M., Muller, A., Kleinfeld, D. \& Tsien, R. Y. ReaChR: a red-shifted variant of channelrhodopsin enables deep transcranial optogenetic excitation. Nat Neurosci 16, 1499-1508 (2013).

34 Rungta, R. L., Osmanski, B. F., Boido, D., Tanter, M. \& Charpak, S. Light controls cerebral blood flow in naive animals. Nat Commun 8, 14191 (2017). 
35 Chaigneau, E. et al. Two-Photon Holographic Stimulation of ReaChR. Front Cell Neurosci 10, 234 (2016).

36 Packer, A. M., Russell, L. E., Dalgleish, H. W. P. \& Häusser, M. Simultaneous all-optical manipulation and recording of neural circuit activity with cellular resolution in vivo. Nature Methods 12, 140-146 (2015).

37 Carrillo-Reid, L., Yang, W., Bando, Y., Peterka, D. S. \& Yuste, R. Imprinting and recalling cortical ensembles. Science 353, 691-694 (2016).

38 Shemesh, O. A. et al. Temporally precise single-cell-resolution optogenetics. Nat Neurosci 20, 1796-1806 (2017).

39 Mardinly, A. R. et al. Precise multimodal optical control of neural ensemble activity. Nat Neurosci 21, 881-893 (2018).

40 Marshel, J. H. et al. Cortical layer-specific critical dynamics triggering perception. Science 365 (2019).

41 Hartmann, D. A. et al. Pericyte structure and distribution in the cerebral cortex revealed by high-resolution imaging of transgenic mice. Neurophotonics 2, 041402 (2015).

42 Chen, I. W., Papagiakoumou, E. \& Emiliani, V. Towards circuit optogenetics. Curr Opin Neurobiol 50, 179-189 (2018).

43 Yang, W. \& Yuste, R. Holographic imaging and photostimulation of neural activity. Curr Opin Neurobiol 50, 211-221 (2018).

44 Rickgauer, J. P. \& Tank, D. W. Two-photon excitation of channelrhodopsin-2 at saturation. Proceedings of the National Academy of Sciences 106, 15025-15030 (2009).

45 Shih, A. Y. et al. The smallest stroke: occlusion of one penetrating vessel leads to infarction and a cognitive deficit. Nat Neurosci 16, 55-63 (2013).

46 Silva, A. C., Lee, S. P., ladecola, C. \& Kim, S. G. Early temporal characteristics of cerebral blood flow and deoxyhemoglobin changes during somatosensory stimulation. $J$ Cereb Blood Flow Metab 20, 201-206 (2000).

47 Hillman, E. M. C. Coupling Mechanism and Significance of the BOLD Signal: A Status Report. Annual Review of Neuroscience 37, 161-181 (2014).

48 Chen, I. W. et al. In Vivo Submillisecond Two-Photon Optogenetics with Temporally Focused Patterned Light. The Journal of neuroscience : the official journal of the Society for Neuroscience 39, 3484-3497 (2019).

49 Yizhar, O. et al. Neocortical excitation/inhibition balance in information processing and social dysfunction. Nature 477, 171-178 (2011).

50 Prakash, R. et al. Two-photon optogenetic toolbox for fast inhibition, excitation and bistable modulation. Nat Methods 9, 1171-1179 (2012).

51 ladecola, C., Yang, G., Ebner, T. J. \& Chen, G. Local and propagated vascular responses evoked by focal synaptic activity in cerebellar cortex. J Neurophysiol 78, 651659 (1997).

52 Chen, B. R., Bouchard, M. B., McCaslin, A. F. H., Burgess, S. A. \& Hillman, E. M. C. High-speed vascular dynamics of the hemodynamic response. Neurolmage 54, 10211030 (2011). 
53 Chen, B. R., Kozberg, M. G., Bouchard, M. B., Shaik, M. A. \& Hillman, E. M. C. A Critical Role for the Vascular Endothelium in Functional Neurovascular Coupling in the Brain. Journal of the American Heart Association 3 (2014).

54 Longden, T. A. et al. Capillary $\mathrm{K}(+)$-sensing initiates retrograde hyperpolarization to increase local cerebral blood flow. Nat Neurosci 20, 717-726 (2017).

55 Zechariah, A. et al. Intercellular Conduction Optimizes Arterial Network Function and Conserves Blood Flow Homeostasis During Cerebrovascular Challenges. Arterioscler Thromb Vasc Biol 40, 733-750 (2020).

56 Drew, P. J. Vascular and neural basis of the BOLD signal. Curr Opin Neurobiol 58, 6169 (2019).

57 Knot, H. J. \& Nelson, M. T. Regulation of arterial diameter and wall [Ca2+] in cerebral arteries of rat by membrane potential and intravascular pressure. J Physiol 508 ( Pt 1), 199-209 (1998).

58 Nystoriak, M. A. et al. Fundamental increase in pressure-dependent constriction of brain parenchymal arterioles from subarachnoid hemorrhage model rats due to membrane depolarization. American Journal of Physiology-Heart and Circulatory Physiology 300, H803-H812 (2011).

59 Cipolla, M. J., Sweet, J. G., Gokina, N. I., White, S. L. \& Nelson, M. T. Mechanisms of Enhanced Basal Tone of Brain Parenchymal Arterioles During Early Postischemic Reperfusion: Role of ET-1-Induced Peroxynitrite Generation. Journal of Cerebral Blood Flow \& Metabolism 33, 1486-1492 (2013).

60 Cipolla, M. J. et al. Increased pressure-induced tone in rat parenchymal arterioles vs. middle cerebral arteries: role of ion channels and calcium sensitivity. J Appl Physiol (1985) 117, 53-59 (2014).

61 Sweeney, P. W., Walker-Samuel, S. \& Shipley, R. J. Insights into cerebral haemodynamics and oxygenation utilising in vivo mural cell imaging and mathematical modelling. Sci Rep 8, 1373 (2018).

62 Kovacs-Oller, T., Ivanova, E., Bianchimano, P. \& Sagdullaev, B. T. The pericyte connectome: spatial precision of neurovascular coupling is driven by selective connectivity maps of pericytes and endothelial cells and is disrupted in diabetes. Cell Discov 6, 39-39 (2020).

63 Hall, C. N. et al. Capillary pericytes regulate cerebral blood flow in health and disease. Nature 508, 55-60 (2014).

64 Itoh, Y. \& Suzuki, N. Control of brain capillary blood flow. Journal of Cerebral Blood Flow \& Metabolism 32, 1167-1176 (2012).

65 Attwell, D., Mishra, A., Hall, C. N., O'Farrell, F. M. \& Dalkara, T. What is a pericyte? J Cereb Blood Flow Metab 36, 451-455 (2016).

66 Gonzales, A. L. et al. Contractile pericytes determine the direction of blood flow at capillary junctions. Proc Natl Acad Sci U S A 117, 27022-27033 (2020).

67 Félétou, M. \& Vanhoutte, P. M. Endothelium-Derived Hyperpolarizing Factor. Arteriosclerosis, Thrombosis, and Vascular Biology 26, 1215-1225 (2006).

68 Tran, C. H. T., Peringod, G. \& Gordon, G. R. Astrocytes Integrate Behavioral State and Vascular Signals during Functional Hyperemia. Neuron 100, 1133-1148.e1133 (2018). 
69 Chuong, A. S. et al. Noninvasive optical inhibition with a red-shifted microbial rhodopsin. Nat Neurosci 17, 1123-1129 (2014).

70 Mohammad, F. et al. Optogenetic inhibition of behavior with anion channelrhodopsins. Nat Methods 14, 271-274 (2017).

71 Mahn, M. et al. High-efficiency optogenetic silencing with soma-targeted anionconducting channelrhodopsins. Nat Commun 9, 4125 (2018).

72 Kim, K. J., Ramiro Diaz, J., Iddings, J. A. \& Filosa, J. A. Vasculo-Neuronal Coupling: Retrograde Vascular Communication to Brain Neurons. The Journal of neuroscience : the official journal of the Society for Neuroscience 36, 12624-12639 (2016).

73 Tong, L. et al. Imaging and optogenetic modulation of vascular mural cells in the live brain. Nat Protoc (2020).

74 O'Herron, P., Summers, P. M., Shih, A. Y., Kara, P. \& Woodward, J. J. In vivo twophoton imaging of neuronal and brain vascular responses in mice chronically exposed to ethanol. Alcohol 85, 41-47 (2020).

75 O'Herron, P. \& von der Heydt, R. Representation of object continuity in the visual cortex. Journal of Vision 11 (2011). 
A

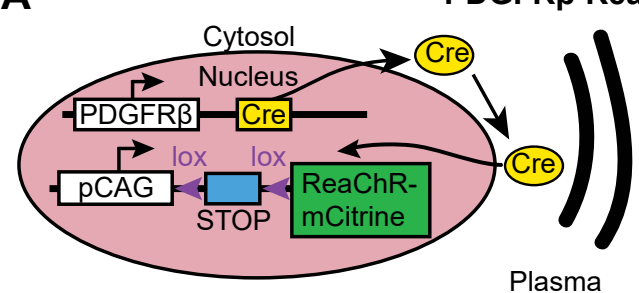

Before Recombination Membrane

PDGFRß-ReaChR-mCitrine Mice

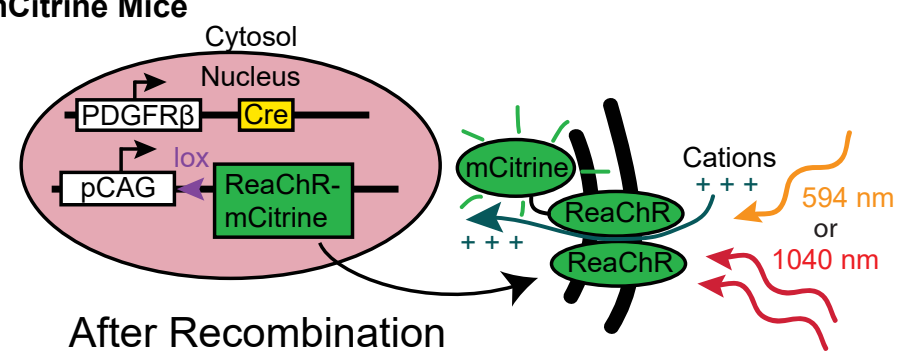

B

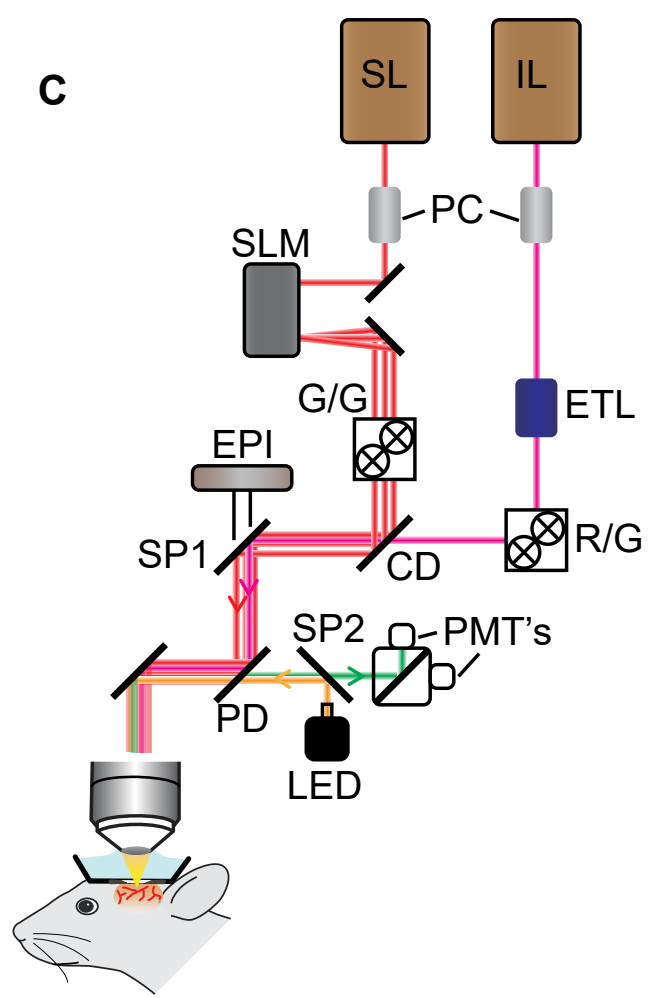

\section{Figure 1. Experimental design: mouse genetics and equipment for controlling arterial diameter with} ReaChR.

A. Crossing the PDGFR $\beta$-Cre line with the ReaChR line leads to expression of the opsin in vascular mural cells. mCitrine is co-expressed as a fluorescent reporter. Mural cells can then be depolarized with single-photons from the $597 \mathrm{~nm}$ LED or two-photons of the fixed $1040 \mathrm{~nm}$ laser. Control mice followed a similar strategy except that instead of mCitrine/ReaChR, YFP was expressed in the cytosol of mural cells. B. Depth projection of cortical vessels from the mouse line created in A. The vascular lumen is labelled with Texas Red dextran and the green in the vessel walls shows the expression of $m$ Citrine/ReaChR. C. Imaging equipment - the Ultima 2P-Plus from Bruker. Two lasers (Imaging laser (IL - tunable from 680-1300 nm) and Stimulation laser (SL - fixed $1040 \mathrm{~nm}$ )) are used for separate imaging and excitation. Laser power is controlled with Pockel Cells (PC). The SL passes through a Spatial Light Modulator (SLM) which is used to create three-dimensional patterns of stimulation light. The spots are then spun in a spiral pattern with a pair of galvonometer mirrors (G/G). The IL can be focused in depth independently of the objective using the Electro-Tunable Lens (ETL). It is scanned over the sample with galvos and/or a resonant scanning mirror $(R / G)$. The two lasers are merged in the light path by the combining dichroic (CD) - a notch filter reflecting light in a narrow band around $1040 \mathrm{~nm}$. The primary dichroic (PD) passes visible light to the detectors while reflecting the infra-red lasers. The LED $(594 \mathrm{~nm})$ is in the visible range so when it is used a short-pass mirror (SP2) must be introduced in the collection path which prevents collection in the red channel of the Photo-multiplier tubes (PMTs). A short-pass mirror (SP1) between the CD and PD allows the use of an epifluorescence module (EPI). In this mode the PD is replaced with a regular mirror. 
bioRxiv preprint doi: https://doi.org/10.1101/2021.01.29.428609; this version posted January 31, 2021. The copyright holder for this preprint (which was not certified by peer review) is the author/funder, who has granted bioRxiv a licensqrigldispliabtoppreprint in perpetuit7wbisphaston

A

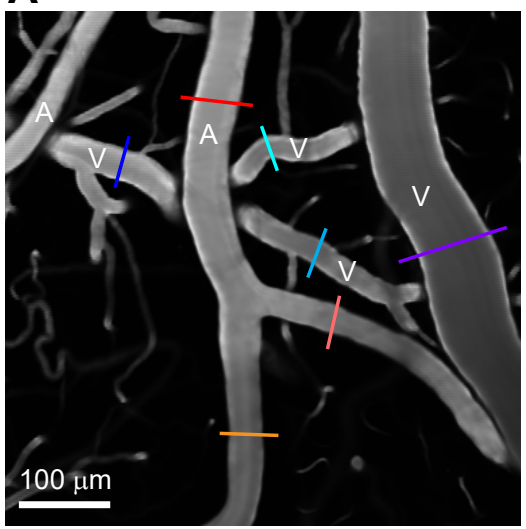

B

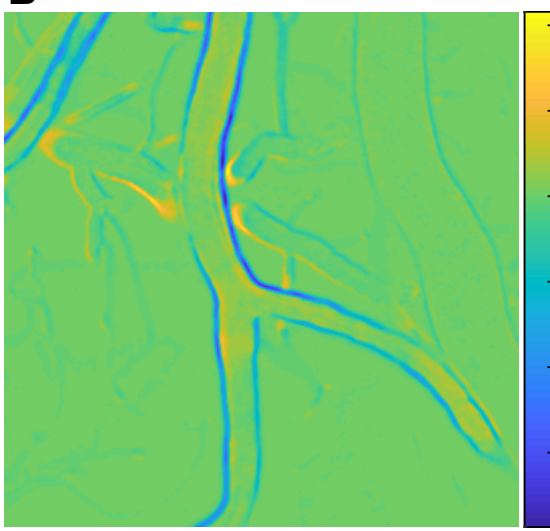
available under aCC-BY-NC-ND 4.0 International license Excitation

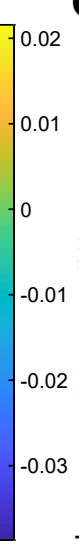

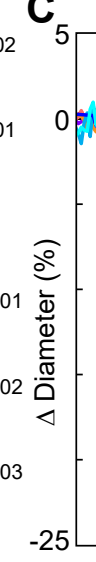

Excitation
D

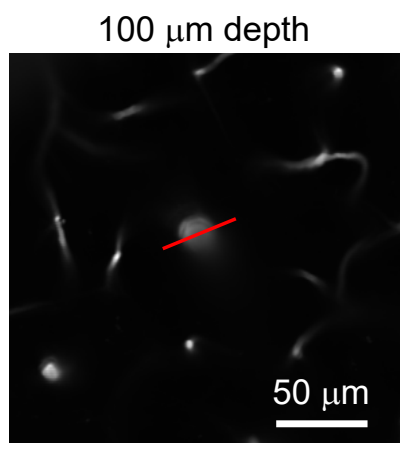

\section{G}

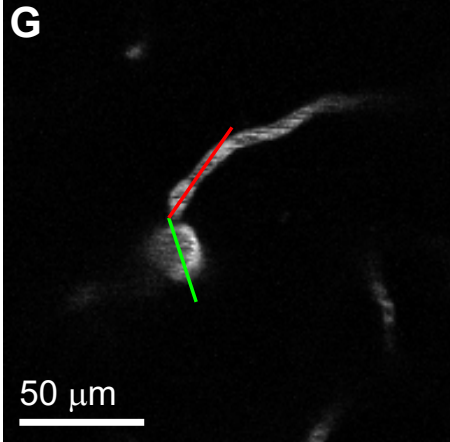

E

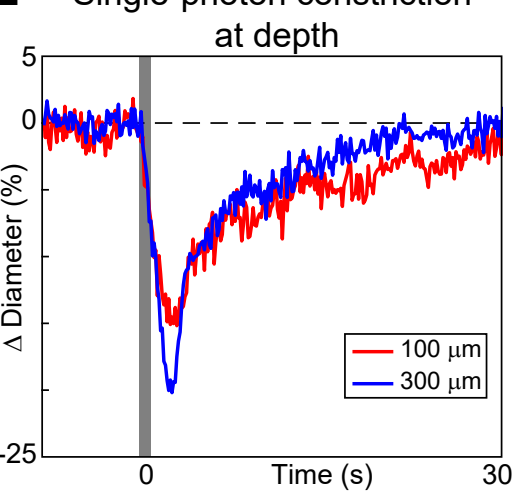

F

H

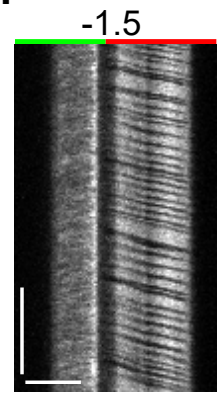

$16.9 ; 430 ; 7.2$
$300 \mu \mathrm{m}$ depth

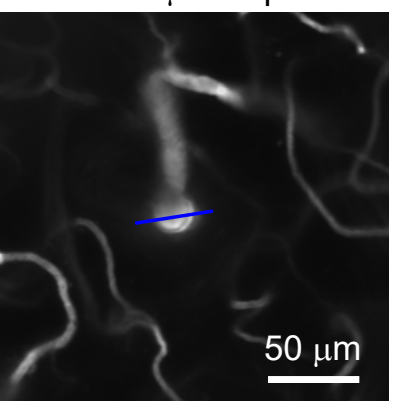

Time
1.5

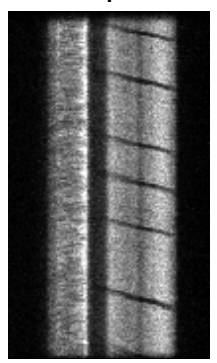

$14.7 ; 60 ; 6.7$

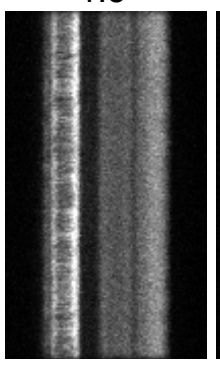

12.5; 0; 0
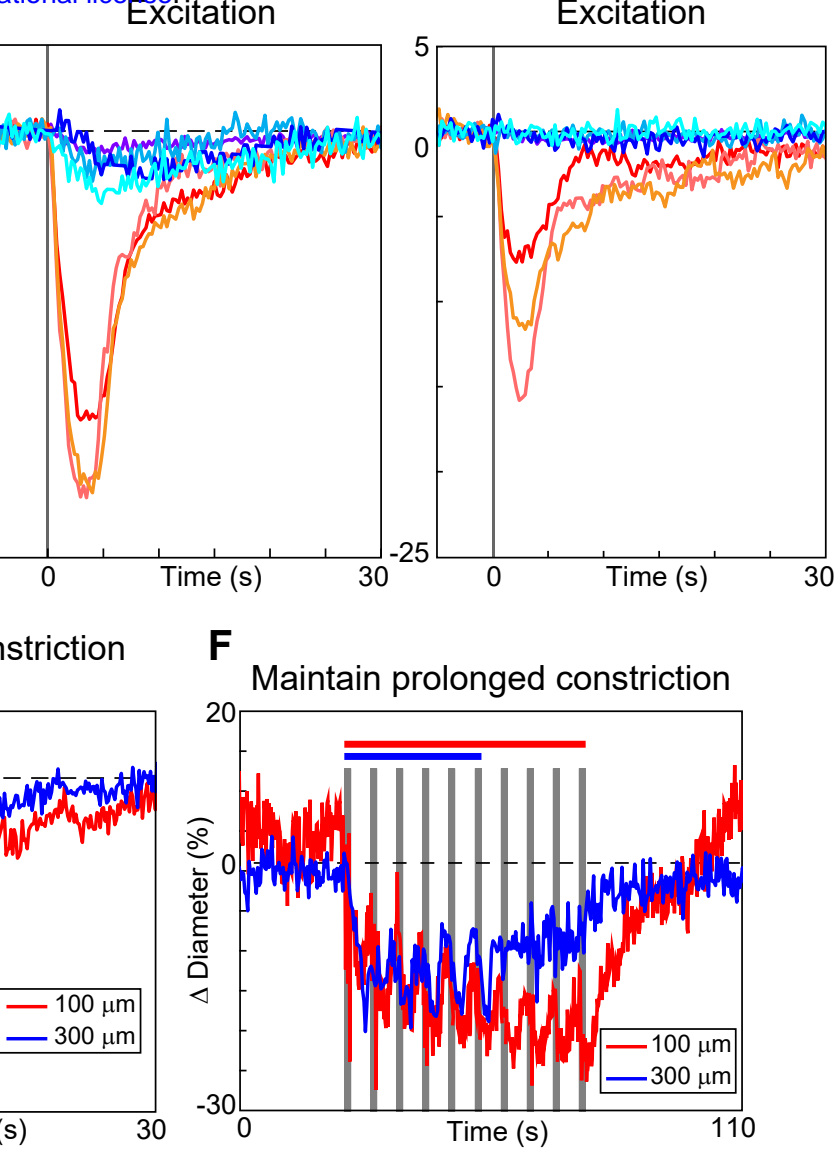

Figure 2. Full-field optogenetic activation.

Diameter $(\mu \mathrm{m})$; Flux (RBCs/sec); Velocity $(\mathrm{mm} / \mathrm{sec})$

2

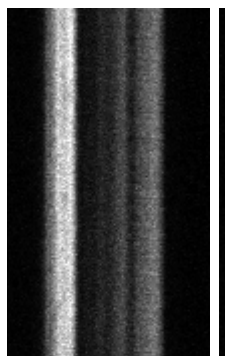

10.3; $0 ; 0$
3.5

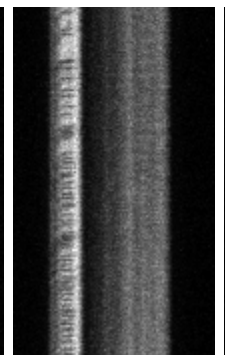

$11.0 ; 0 ; 0$
4.5

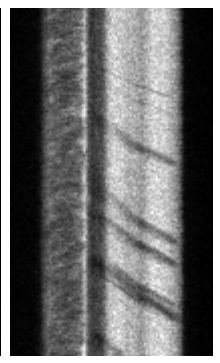

$16.1 ; 60 ; 2.5$
6.5

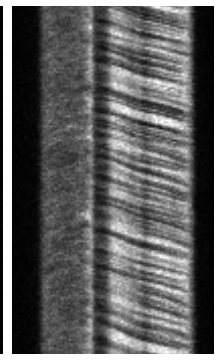

$18.3 ; 400 ; 5.2$

A. Cortical surface vessels labelled with FITC dextran. Arteries (A) and veins (V) are marked. B. Difference image created by subtracting the average of 4 seconds of imaging following stimulation (2.5 - 6.5 seconds after stimulation onset) from the average before stimulation (4 seconds before onset). Blue lines on vessel walls opposite each other indicate reduced brightness showing where constriction occured. Vessels with yellow on one side and blue on the other indicate XY shifts in the image between the two intervals. Units are difference in arbitrary brightness values. C. Time course of vessel diameter. Colored lines correspond to cross-sections in A. Because veins showed small constriction to the full-field (single-photon) optogenetic stimulation (left panel), we also tested the vessels with two-photon stimulation targeted to one vessel segment at a time at the cross-section locations (right panel). Vertical gray band is optical stimulation interval (100 ms pulse). Arteries were stimulated with $\sim 110 \mathrm{~mW}$ total power. Veins were stimulated with up to $\sim 330 \mathrm{~mW}$. All stimulations were 2 adjacent spots on each side of the vessel (4 total), $25 \mu \mathrm{m}$ diameter each, centered at the drawn cross-sections. A-C: representative data from 3 mice. D. Images of penetrating arterioles $100 \mu \mathrm{m}$ deep (left) and $300 \mu \mathrm{m}$ deep (right) in the cortex. Colored cross-sections correspond to time course traces in E and F. E. A single train of LED light pulses (5 pulses, $100 \mathrm{~ms}$, with $100 \mathrm{~ms}$ between) evoked strong constriction at both depths. F. We maintained vasoconstriction with repeated stimulations trains ( 5 pulses, $100 \mathrm{~ms}$, with $100 \mathrm{~ms}$ between, 4 seconds between trains). 6 trains were applied to the vessel at $300 \mu \mathrm{m}$ and 10 to the vessel at $100 \mu \mathrm{m}$. D-F: representative data from 5 mice. G. Image of penetrating arteriole with a daughter branch $85 \mu \mathrm{m}$ below the surface. Line scans were acquired to measure the diameter of the arteriole (green segment) and the flow of the daughter branch (red segment). A train of pulses (10 pulses, $100 \mathrm{~ms}$, with $100 \mathrm{~ms}$ between) was delivered. $\mathbf{H}$. Line scan data from 7 time points around the stimulation train. Y-axis is time with each row being a single scan of the laser line. $\mathrm{X}$-axis is distance along the line. The left part of each panel shows the diameter data (the green segment) and the right part shows the flow (the red segment). The dark streaks indicate red blood cells passing through the vessel. Numbers below each panel give the diameter of the penetrating arteriole and the flux and blood velocity for the daughter branch. Flow is reduced in the daughter branch and then drops to zero (absence of dark streaks) before slowly recovering. The arteriole constricts and flow is briefly eliminated (absence of dark spots in diameter segment at 2 seconds) before returing as the arteriole dilates back to the baseline level. G-H: representative data from 3 mice. Scale bars: Time axis: $25 \mathrm{~ms}$; Distance axis: $25 \mu \mathrm{m}$. 
bioRxiv preprint doi: https://doi.org/10.1101/2021.01.29.428609; this version posted January 31, 2021. The copyright holder for this preprint (which was not certified by peer review) is the author/funder, who has granted bioRxiv a license to display the preprint in perpetuity. It is made

A

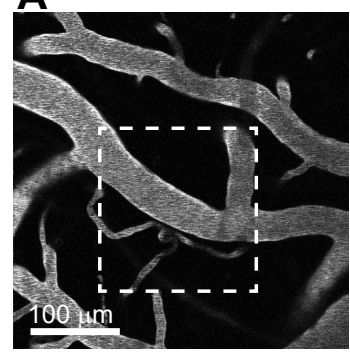

B available-under aCC-BY-NC-ND 4.0 International license.
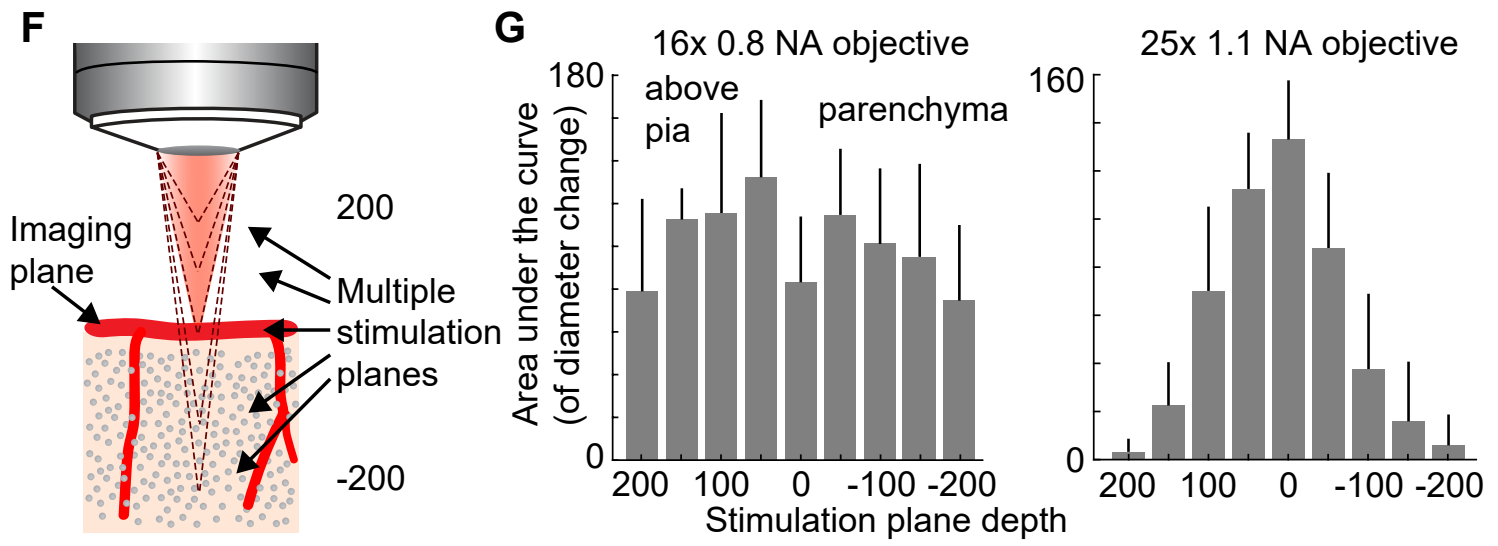

E

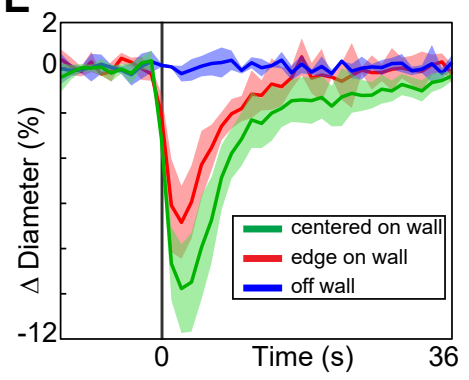

Stimulate site 1

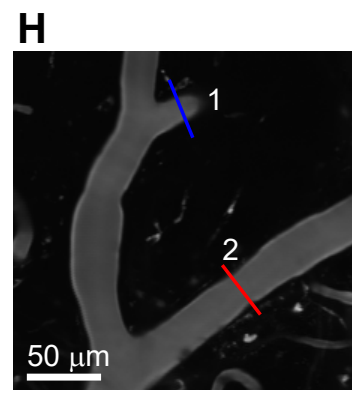

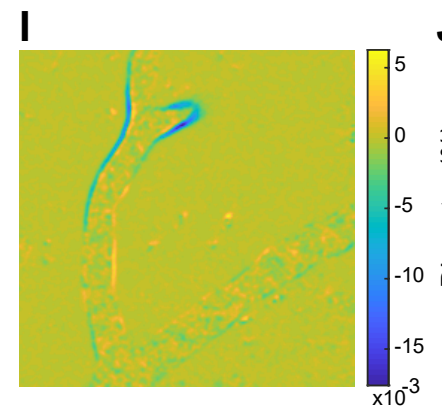

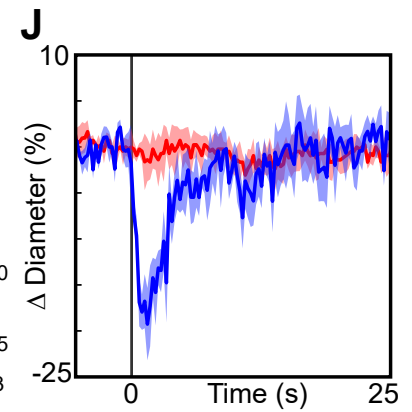

Stimulate site 2

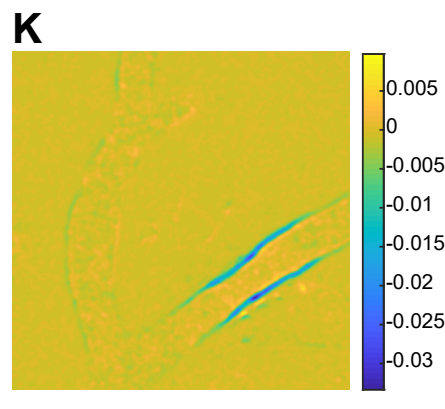

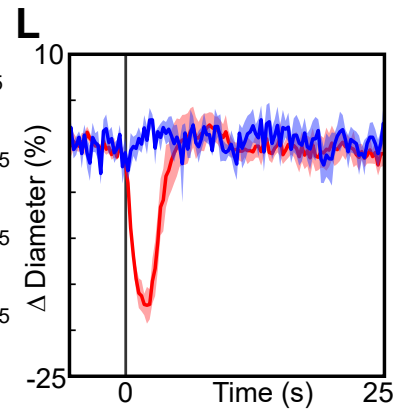

Figure 3. Spatial precision of two-photon optogenetic activation.

A-E. XY-precision of photostimulation. A. Image of surface vessels in an opsin mouse that were photostimulated with spots in different positions relative to the vessel wall. Dashed rectangle indicates region in inset on the difference image panels (B-D). Three spot positions were used: B - spots centered on vessel walls, C - spot edges on vessel walls, and D - spot centers one diameter away from vessel walls. Difference images as in Figure 2. Again, dark bands on vessel edges indicate constriction. Red circles indicate the location and size of the spots, white asterisks indicate spot centers. E. Time course of diameter change based on spot position. Average of three vessels where this manipulation was performed, with 5 repetitions for each vessel. Error bands are standard deviation. A-E used the Nikon 16x 0.8 N.A. objective F-H. Z-precision of photostimulation. F. Scematic of imaging setup with the objective focusing the imaging beam on a pial artery sitting on the cortical surface. The dashed lines indicate various focal planes of the stimulation laser generated by the SLM. In the experiment, stimulation spots were focused from $200 \mu \mathrm{m}$ above to $200 \mu \mathrm{m}$ below the imaging plane in $50 \mu \mathrm{m}$ steps. 2 or 3 adjacent pairs of $25 \mu \mathrm{m}$ diameter spots (4 or 6 total) were placed on or directly above or below a vessel segment. Total spot power in both experiments was $\sim 115 \mathrm{~mW}$. G. Constriction amount at the different depths computed as the average area under the curve across repetitions for the first 5 seconds following the stimulation pulse. Error bars are standard deviation across 8 repetitions. Left; results from a $40 \mu \mathrm{m}$ diameter surface artery using the Nikon $16 x$ objective. Right; results from a $38 \mu \mathrm{m}$ diameter surface artery using the $25 x$ objective. H-L. Stimulation of different surface branches leads to isolated constriction. $\mathbf{H}$. Anatomical image of surface vasculature labelled with Texas Red dextran. Colored lines indicate cross-sections for time course plots in K,M. Stimulation spots were centered at the location of the lines in two separate runs. I,K. Difference images showing constriction (blue lines on both sides of vessel) only near stimulation points. J,L. Time course of diameter for the two different stimulations: error bands are standard deviation across five $(\mathrm{J})$ or four $(\mathrm{L})$ repetitions. Vertical gray band is stimulation interval $(100 \mathrm{~ms})$. $\mathrm{H}-\mathrm{L}$ : representative data from 10 mice. 
A

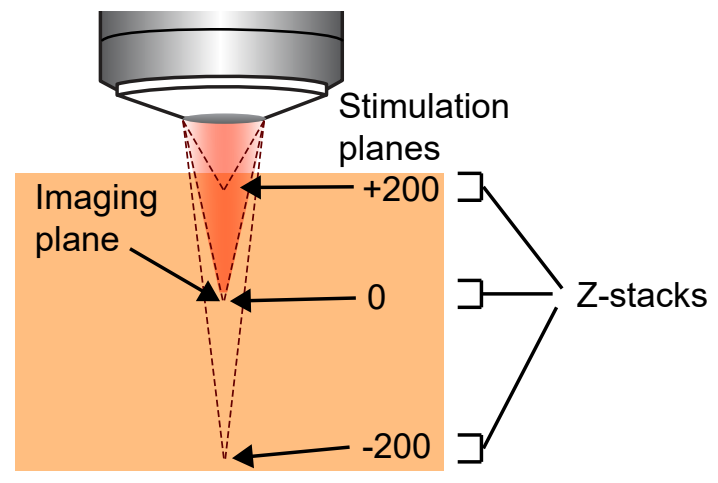

B Photobleaching fluorescent slide with SLM

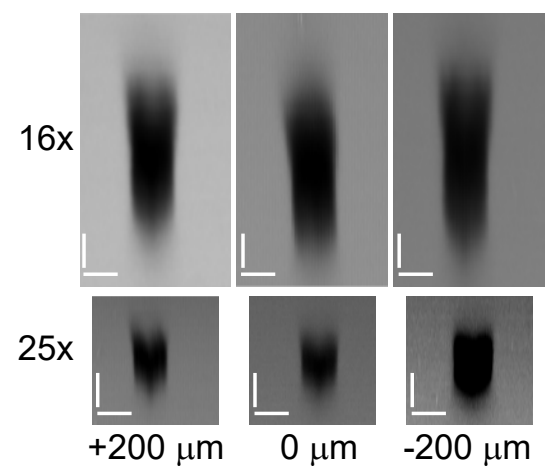

Figure 3-1. Axial spread of SLM stimulation with different objectives.

A. Scematic of imaging setup with the objective focusing the imaging beam in the middle of a fluorescent Chroma slide. The dashed lines indicate the three focal planes of the stimulation laser generated by the SLM. Stimulation spots ( $15 \mu \mathrm{m}$ diameter) were focused $200 \mu \mathrm{m}$ above, $200 \mu \mathrm{m}$ below, or at the imaging plane and high powers were delivered to bleach the fluorophore in the slide. Z-stacks were subsequently obtained with the imaging laser around each of the stimulation depths to determine the spread of the bleaching (and hence the spread of photo-stimulation). H. XZ-projections from the fluorescent slide. The spread of bleaching in the axial $(Z)$ dimension was the same for a given objective regardless of the SLM focus depth, indicating that the SLM was functioning properly. Scale bars are $15 \mu \mathrm{m}$ for both the axial and lateral dimensions. 

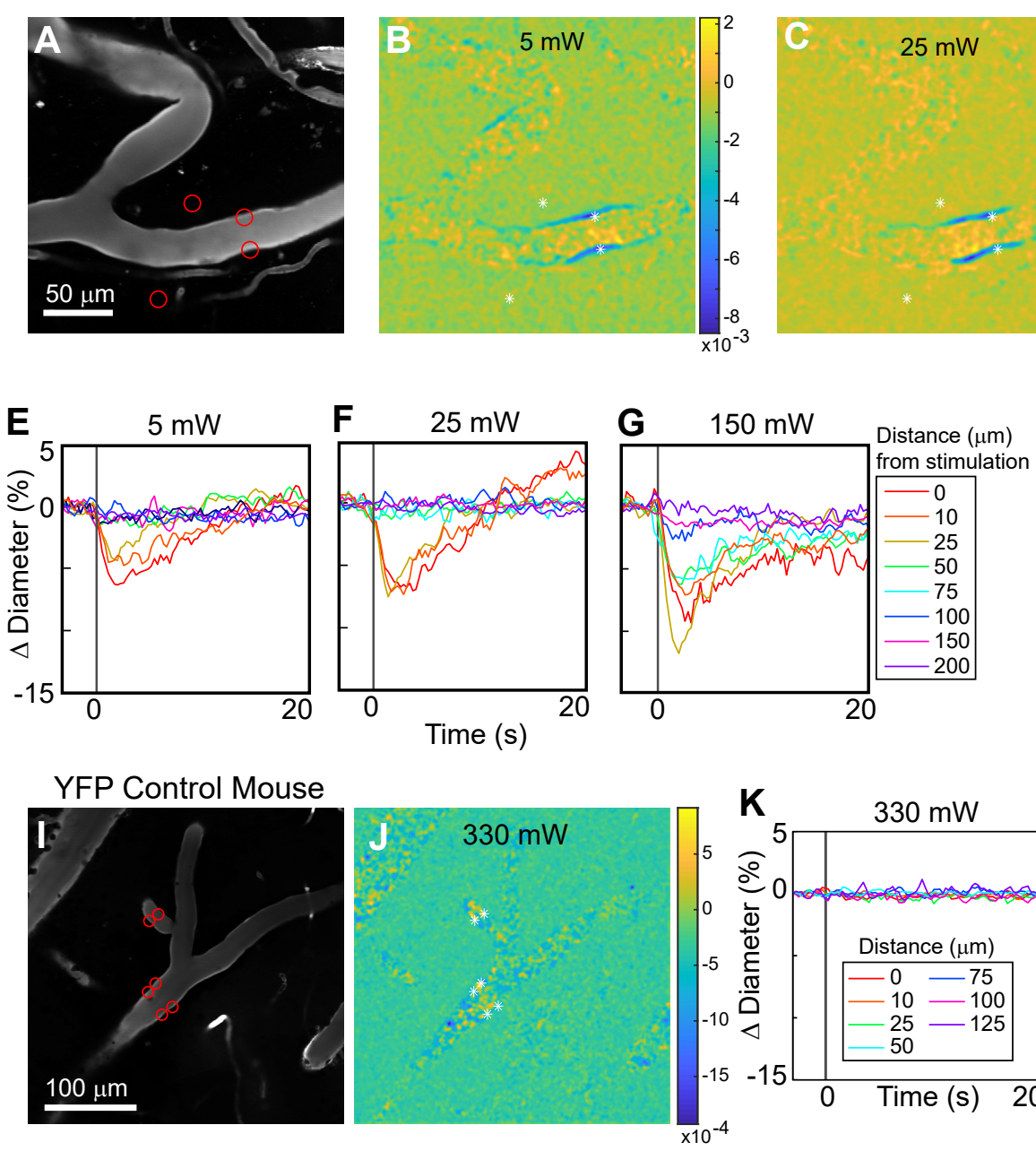
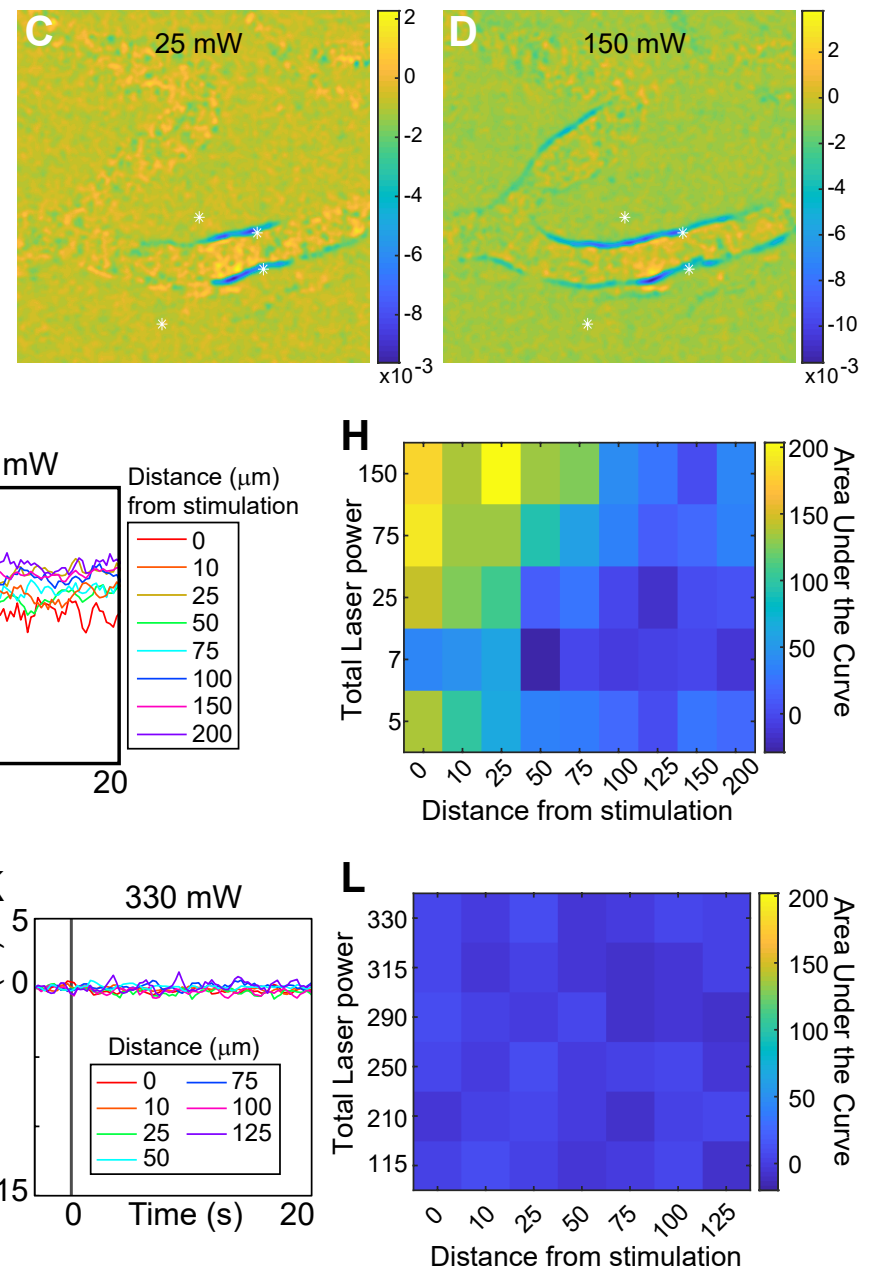

\section{Figure 4. Controls for laser power.}

A. Vessels labelled with Texas Red dextran. Red circles show spot size and location. Two spots off the vessel were to maintain similar power levels per spot when only two spots were placed on the vessel. B-D. Difference images (as in earlier figures) for three different power levels (values indicate total power of all 4 spots). E-G. Time courses of diameter change at the three power levels in B-D at different distances from the center of stimulation. H. Summary of constriction at all power levels tested across different distances. Area under the curve is the absolute value of the sum of the data points over 8 seconds beginning at the stimulation onset (higher numbers/yellow color indicate greater constriction). A-H: representative example from 6 vessels in 3 mice. I-L. Same but for a control mouse expressing YFP but no opsin in mural cells. Difference map $(\mathrm{J})$ and time course $(\mathrm{K})$ are only shown for highest power. Time course $(\mathrm{K})$ is for cross-section on segment with 4 spots. Even at very high powers, no constriction was seen. Represenatative example from 12 vessels in 2 mice. 


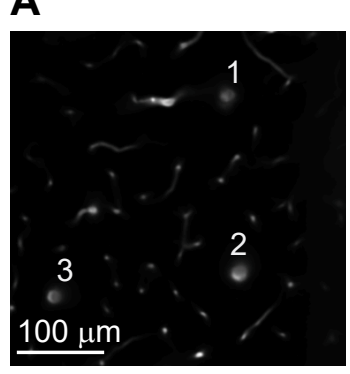

B
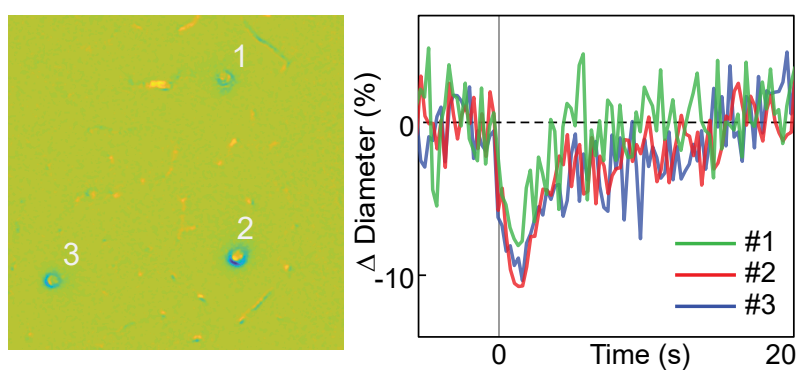

C

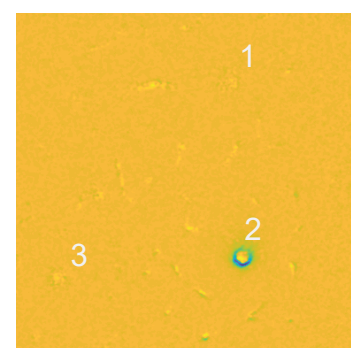

Stimulate vessel \#2

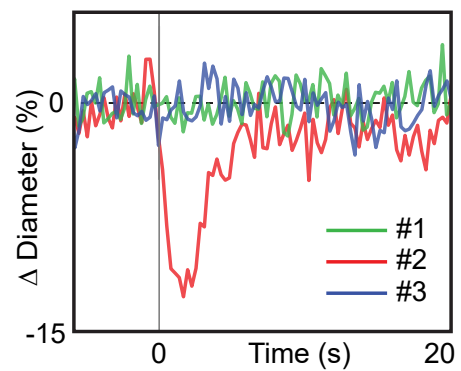

\section{Stimulate $170 \mu \mathrm{m}$ below surface}

D

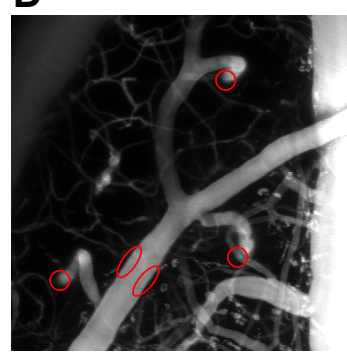

E

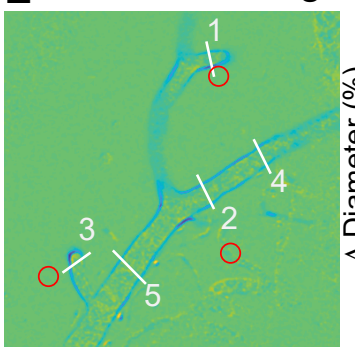

Image at surface

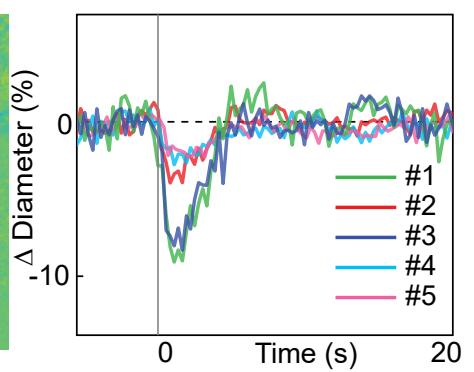

F Image $300 \mu \mathrm{m}$ below surface

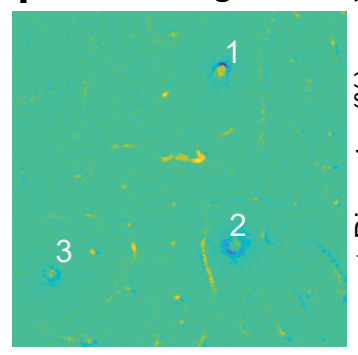

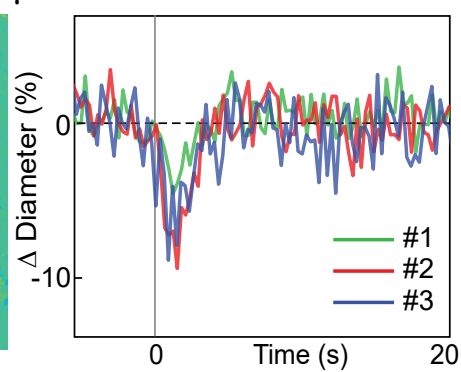

\section{G Stimulate and image at surface}
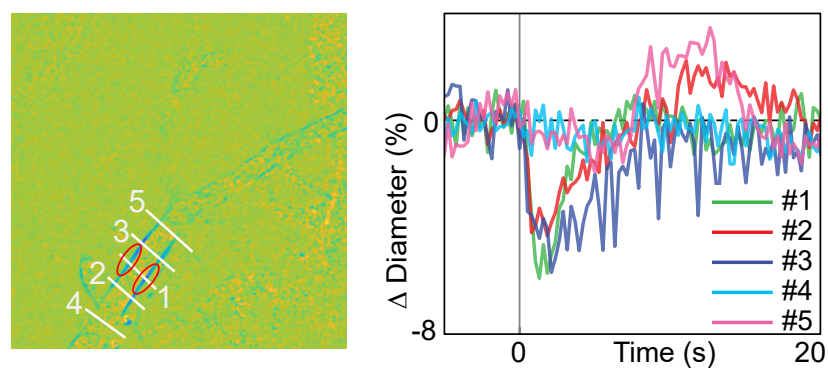

Figure 5. Single-vessel precision and spread of activation for sub-surface stimulation.

A. Three penetrating arterioles visualized at $170 \mu \mathrm{m}$ below the cortical surface - vasculature labelled with Texas Red dextran. B-C. Stimulation and imaging are performed at the same $170 \mu \mathrm{m}$ depth plane either in all three vessels (B) or just in vessel \#2 (C). Left panels are difference images as in earlier figures. Right panels are time courses of diameter changes of the vessels indicated in the left panels. D. The same imaging location but focused on the cortical surface. The red circles indicate the location of the three penetrating arterioles at the stimulation plane $170 \mu \mathrm{m}$ below the surface. The red ovals indicate the span of stimulation spots ( 3 adjacent spots on either side of the vessel, $12 \mu \mathrm{m}$ diameter) for the surface stimulation in panel G. E-F. Stimulating $170 \mu \mathrm{m}$ below the surface while imaging the cortical surface $(E)$ or $300 \mu \mathrm{m}$ below the cortical surface (F). For B,E,F, the total laser power divided between the three vessels was $\sim 300 \mathrm{~mW}$. For C, the total power of $\sim 300 \mathrm{~mW}$ was divided between 3 spots on vessel 2 and 2 off-target spots at different locations in the tissue to reduce the overall power at vessel 2. G. Stimulating and imaging at the cortical surface. Total laser power across the 6 spots on the surface vessel was $\sim 180 \mathrm{~mW}$. All images panels follow the scale in A. Data was collected with the Nikon 25x objective. Representative data from 4 mice. 
A

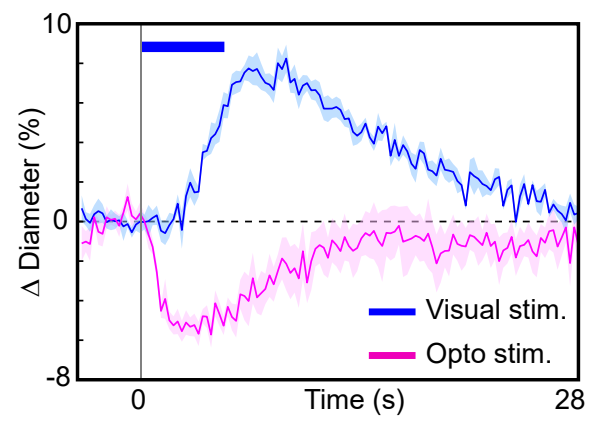

C

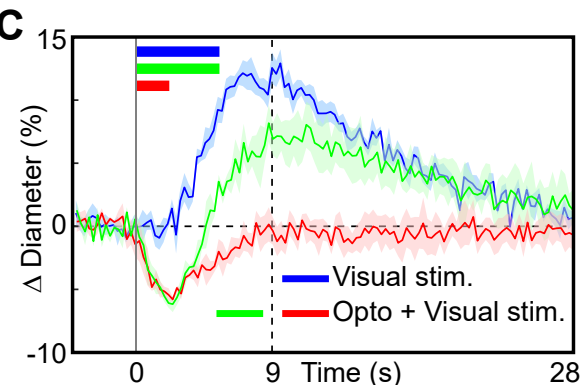

B

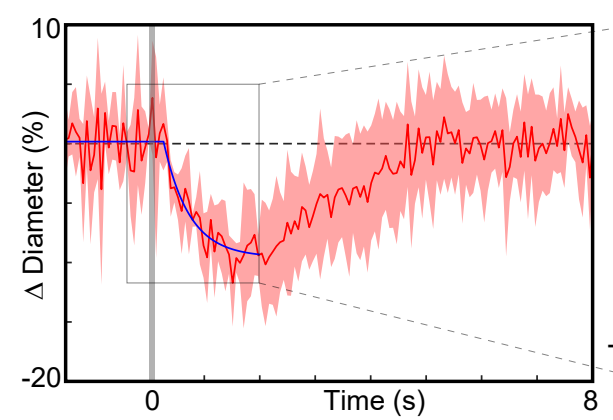

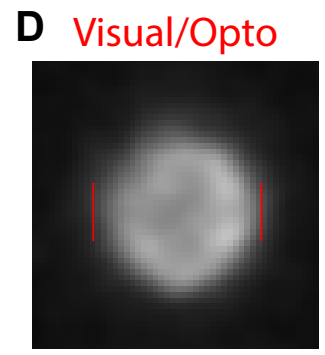

$14.8 \mu \mathrm{m}$

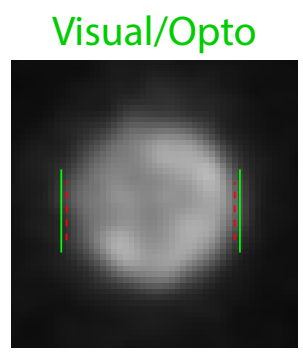

$15.7 \mu \mathrm{m}$
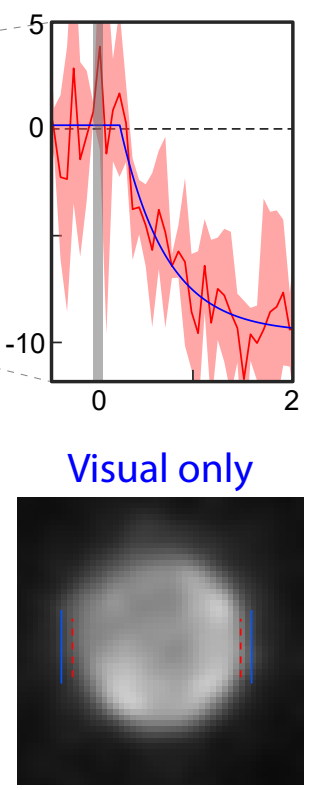

$16.8 \mu \mathrm{m}$

Figure 6. Optogenetic stimulation and sensory stimulation.

A. Time course of dilation of a penetrating arteriole to visual stimulation (blue) and of constriction of the same artery to optogenetic stimulation (magenta). Vertical gray band is optogenetic stimulation interval $(100 \mathrm{~ms})$. Blue bar is visual stimulation interval ( 5 seconds). B. Time course of average of 5 arteries that were imaged at high speed $(15 \mathrm{~Hz})$ to determine onset latency. Error band is standard deviation across runs. Blue curve is the fit to the data. A constant and an exponential curve were fit with the time of the switch between the two giving the onset latency (see Methods). Vertical gray band is $100 \mathrm{~ms}$ light pulse. Right panel: Zoomed in view of plot around onset time for better visualization. C. Pairing optogenetic stimulation with visual stimulation leads to reduced (green) or elimininated (red) visually evoked dilations compared to visual stimulation alone (blue). Vertical gray band at time 0 is optogenetic stimulation interval (100 ms) and colored bars indicate visual stimulation duration (blue and green: $5 \mathrm{sec}$; red: $2 \mathrm{sec}$ ). Vertical dashed line at 9 seconds indicates time point of images shown in D. D. Single-frame images showing penetrating arteriole 9 seconds after stimulus onset for the three conditions shown in $\mathrm{C}$. Colored lines and values indicate the diameter of the automated fit computed for the frames. Colors correspond to traces shown in C. Red dashed line in middle and right panels are the fit from the left panel superimposed for comparison purposes. A,C-D: representative examples from 10 mice. 


\section{Movie Legends}

Movie 1. Full-field activation with the $594 \mathrm{~nm}$ LED in a PDGFR $\beta /$ ReaChR mouse. Image is looking down at pial surface vessels in the neocortex labelled with FITC dextran. A single 100 ms pulse from the $549 \mathrm{~nm}$ LED was used to stimulate the vessels. In this and other movies, the dark flash indicates the time of the optical stimulation. The image darkens because the detectors are briefly blocked by mechanical shutters to protect them from the intense stimulation light. This and subsequent movies are presented at $4 \mathrm{x}$ real time.

Movie 2. Constriction of a penetrating arteriole with the $594 \mathrm{~nm}$ LED. 5 pulses, $100 \mathrm{~ms}$ duration with $100 \mathrm{~ms}$ in between were presented.

Movie 3. The same vessel as Movie 3 was stimulated with 10 sets of 5 pulses, with 4 seconds between the onset of the pulse trains. Power and duration of pulses as in Movie 2.

Movie 4. Two-photon activation leads to focal constriction. The SLM focused 6 spots at the location indicated by the red arrow. The total stimulation power was $\sim 200 \mathrm{~mW}$ spread over the 6 spots with 3 adjacent spots on either side of the vessel. Each spot was scanned in a $12 \mu \mathrm{m}$ diameter spiral for $100 \mathrm{~ms}$. The white arrow is for comparison with Movie 5 . Vessels are labelled with Texas Red Dextran.

Movie 5. The same field-of-view as Movie 4 but here a different branch of the pial artery is stimulated (red arrow). Total power was $\sim 75 \mathrm{~mW}$ spread out over 3 spots positioned on the branch stub and 3 off target spots. The white arrow indicates the location stimulated in Movie 4 for comparison.

Movie 6. Excessive laser power leads to vessel damage and widespread constriction. Same vessel as Figure 4A, here using a total of $\sim 230 \mathrm{~mW}$ of laser power. The first pulse leads to a visibly damaged vessel wall and the second pulse breaks the wall causing dye leakage.

Movie 7. Control mouse showing no constriction after light pulse when using powers that resulted in clear constrictions in ReaChR mice. The SLM focused 4 total spots, with pairs of spots opposite each other at the two different positions on the artery indicated by the arrows. The total stimulation power was $\sim 200 \mathrm{~mW}$, and as before, each spot was scanned in a $12 \mu \mathrm{m}$ diameter spiral for $100 \mathrm{~ms}$.

Movie 8. Excessive laser power in control animals can lead to widespread constriction. Same as Movie 7 but the power was increased to $\sim 265 \mathrm{~mW}$.

Movie 9. A second example vessel from a control mouse stimulated with a total laser power of $\sim 200 \mathrm{~mW}$ spread over 4 spots (pairs of spots opposite each other at arrow locations).

Movie 10. Same as Movie 9 but the power was increased to $\sim 280 \mathrm{~mW}$. 